\title{
Tactile Discrimination of Shape: Responses of Slowly and Rapidly Adapting Mechanoreceptive Afferents to a Step Indented into the Monkey Fingerpad
}

\author{
Mandayam A. Srinivasan and Robert H. LaMotte \\ Department of Anesthesiology, Yale University School of Medicine, New Haven, Connecticut 06510
}

The representation of shape in the responses of monkey cutaneous mechanoreceptive afferents to steps of varying shape vertically indented into the fingerpad was studied. A series of flat plates was used, each with a step change in thickness in the middle so that one-half of the plate was thicker than the other. The cross-sectional shape of the step approximated that of a half-cycle sinusoid, $0.5 \mathrm{~mm}$ high, that was varied in half-cycle wavelength (step width) and hence in steepness and curvature. The steps fell into 2 categories, characterized as "steep" and "gradual." Evoked action potentials were recorded from single, slowly adapting and from rapidly adapting Meissner corpuscle mechanoreceptive afferent fibers (SA and RA, respectively) innervating the fingerpad of the anesthetized monkey while each step was indented at a succession of lateral positions across the fiber's receptive field.

The responses of each SA provided a spatial response profile (number of evoked impulses as a function of step position) that was directly related to the variation in curvature across the step. The rate of discharge was greatest under the sharpest (convex) portion of the step, least under the adjacent concave portion, and intermediate under the flat portions of the steps. The results indicated an exquisite sensitivity of the SA, even during the ramp phase of vertical indentation, to the changes in skin curvature. The spatial response profile remained relatively undistorted over time during the ensuing steady phase, while the contrast between the peak and the minimum response improved. RAs responded only during the ramp phase and with fewer responses, and gave rise to a poorly modulated spatial response profile, even though half of the RAs tested showed limited sensitivity to the amount or rate of change of skin curvature. It was hypothesized that RA responses are predominantly influenced by the vertical velocity of the most sensitive spot in the receptive field. When the same step stimuli were applied to the human fingerpad, the capacities of humans to discriminate differences in step shape were found to correlate with the discriminability of SAs, as opposed to the considerably poorer discriminability of RAs. It

Received June 16, 1986; revised Nov. 19, 1986; accepted Jan. 8, 1987.

We thank James Whitehouse for his assistance in all phases of the study, David Keddy for computer programs, and Laurie Hauer for typing the manuscript. This research was supported by PHS Grant 15888.

Correspondence should be addressed to Robert H. LaMotte, Ph.D., Department of Anesthesiology, Yale University of Medicine, 333 Cedar Street, New Haven, CT 06510.

Copyright @ 1987 Society for Neuroscience $0270-6474 / 87 / 061682-16 \$ 02.00 / 0$ is concluded that information concerning the local curvature and hence the shape of objects indenting the skin is primarily coded by the SAs.

In the 2 preceding papers (LaMotte and Srinivasan, 1987a, b), we investigated the responses of SAs and RAs to the same sinusoidal steps stroked back and forth across the fingerpad under constant compressional force. The discharge rates of RAs responding to the sinusoidal portions of the steps were superior to those of SAs in conveying "intensive" information related to the sharpness of steps under stroking. Because of their excellent sensitivity to skin curvature, only SAs provided spatial information about tactile shape-for example, its curvature profile-under both stimulus conditions of stroking a step across and statically indenting it into the skin. An implication of these findings is that the central nervous system has direct access to a feature of the object's surface shape, its curvature distribution, that remains invariant with respect to translation and rotation of the object.

Primates use predominantly their fingerpads for the exploration of local variations in the shapes and surface textures of objects through tactile sensing. It is known that both the highest density of mechanoreceptors and the greatest capacity for spatial resolution in the hand are present in the fingerpads (Johansson and Vallbo, 1979; Darian-Smith and Kenins, 1980). With any single static contact, tactile information about only that region of the surface of the object that lies within the area of contact with the skin, or "contact oval," can be obtained, and the size of the contact oval depends on the mechanical properties of the object and the finger, as well as the forces used. In the present study, we consider only objects that can be treated as rigid, their surface deformations caused by the application of the finger being negligible. Owing to the finger size and the usual forces exerted by humans (15-90 gm wt; see LaMotte and Srinivasan, 1987a), the depth of skin indentation is typically 1 or $2 \mathrm{~mm}$ and the width, or "diameter," of the area of contact about 7-12 mm. Therefore, a single static contact provides information about the object's surface only up to a size of the order of millimeters. However, successive shifts of the contact oval over the object's surface enable even bigger shapes to be perceived, with the central nervous system integrating both the cutaneous and proprioceptive information obtained over time. We are, at present, interested in the peripheral neural representation of the shapes of rigid objects that are of the size of a few fingerpad contact ovals.

The geometrical description of any rigid object in 3-dimensional space is complete if its position and orientation with 
respect to a chosen coordinate frame is specified in addition to its shape. By "shape" we mean that property of its surface that remains invariant with the translation and rotation of the object as a rigid body. The surface feature that satisfies this property is the curvature at any point, defined as the reciprocal of the radius of the circle that can be fitted locally to the surface profile on a given cross section. It is well known from differential geometry that the shape of an objcct is completely specified if its curvatures are known at every point on its surface. Thus, perception of the shape of an object independent of its position and orientation in 3-dimensional space requires a knowledge of the distribution of curvatures on its surface.

Two primary modes of gathering tactile information about the surface of a stationary object consist of pressing the fingerpad onto the object and either holding the fingerpad stationary or stroking it across the surface of the object. Stationary vertical indentation is a much simpler stimulus than is lateral movement because of the synchronous motion of each point of the skin in contact with the object and the virtual absence of horizontal stretch. While both the rapidly and slowly adapting mechanoreceptors within and in the neighborhood of the contact oval respond during the ramp phase of vertical indentation, only the slowly adapting mechanoreceptors respond when the force or depth of indentation is maintaincd as constant.

Previous studies involving the classification of mechanoreceptive afferent fibers using vertical indentations with blunt probes (Knibestöl, 1973, 1975; Pubols, 1982a; Pubols and Pubols, 1983) have typically varied the force, depth, and velocity of indentation, but not the shape of the probe. Recently, objects whose surfaces had spatially organized stimuli, such as braillesized dot patterns, have been stroked across or applied statically to the skin (Darian-Smith and Oke, 1980; Darian-Smith et al., 1980; Johnson and Lamb, 1981; Lamb, 1983). The general conclusion of these studies was that only the responses of slowly adapting fibers (SAs) accounted for the tactile spatial resolution achieved by humans. The responses of the Meissner corpuscle type of rapidly adapting fibers (RAs), as well as those of Pacinian fibers, were much poorer in spatial detail. In a study in which gratings consisting of bars and gaps were applied statically to the monkey's fingerpad, it was found that the SAs gave a much greater response to the edge of a bar placed on the most sensitive spot in their receptive fields than to the flat part of the bar (Phillips and Johnson, 1981a). Using objects whose surfaces had sharp edges, other investigators have also observed that the SAs are particularly sensitive to edges, while the RAs are generally poor in their edge sensitivity (Vierck, 1979; Johansson et al., 1982).

A more general hypothesis, namely, that the SAs are sensitive to changes in the curvature of the skin surface at the most sensitive spot within their receptive fields, is entirely consistent with the experimental results mentioned above. This does not mean that the "bending" of the receptor is the cause of the enhanced response, but that the sharp edges applied to the most sensitive spot cause a higher change in skin surface curvature than a flat stimulus does, resulting in higher stresses and strains at the receptor site, which, in turn, elicit a higher response. In order to explore this idea further, we chose to work with simple, smooth surfaces with variable curvatures. Each of these consisted of a step change in the thickness of a flat plate, making one-half of the plate thicker than the other. The cross-sectional shape of the region of transition in thickness approximated a half-cycle of sinusoid. The wavelength of the sinusoid (width of the step) was varied while keeping the step height constant. Thus, the step shapes had the same form of curvature profile (i.e., sinusoidal), but differed in peak curvature and wavelength.

In the 2 preceding papers (LaMotte and Srinivasan, 1987a, b), we investigated the evoked responses of SAs and RAs when the sinusoidal steps were stroked laterally while the total vertical force on the finger was kept constant. Two hypotheses were proposed to provide a consistent interpretation of all the major features of the afferent responses: SAs are highly sensitive to the amount and rate of change in skin curvature at the most sensitive spot in their receptive fields, while RAs are predominantly influenced by the vertical velocity of the skin at that spot. In this paper, we describe the responses of the SAs and the RAs to the vertical indentation of the same sinusoidal steps and demonstrate that the same hypotheses remain valid. There is a major difference between the responses of the populations of SAs and RAs to stroking and the responses to stationary vertical indentation. During stroking, the population response at every instant of time is composed of both the SA and RA responses. However, under stationary vertical indentation, both the SAs and RAs respond during the initial ramp phase of indentation, whereas during the ensuing steady phase, the RAs become silent and only the SAs continue to encode the stimulus shapc.

\section{Materials and Methods}

Shapes of the steps. Transparent epoxy plates $(20 \times 45 \mathrm{~mm})$ were used to indent the finger. One surface of each of these plates was flat, while the other contained a single smooth step that, in cross section, was shaped as a half-cycle of a sinusoid (see inset in Fig. 4). [For details about the construction of these steps and their shapes drawn to an enlarged scale, refer to LaMotte and Srinivasan (1987a).] The shape of the step was characterized by the spatial variation in curvature (curvature distribution). For all the steps the form of the distribution was sinusoidal. The magnitude of the curvature distributions of the steps was varied by fixing the step height at $0.5 \mathrm{~mm}$, while varying the step "width," or half-cycle wavelength. Six steps were used, 5 smooth sinusoids and 1 containing sharp right-angle corners. The half-cycle wavelengths used were $0,0.450,1.195,1.230,3.038$, and $3.134 \mathrm{~mm}$, and these steps were consecutively numbered from 0 to 5 . Steps $0-3$ were described as feeling "steep," and the other 2 as "gradual" by human subjects.

Applying the steps to the passive fingerpad. A servocontrolled mechanical stimulator (LaMotte et al., 1983) was used to displace the plate containing a step along vertical and horizonlal axes. A given experimental run with each step consisted of a series of trials. During each trial, a plate containing the step was brought down vertically, with constant velocity, onto the skin (usually at $4 \mathrm{~mm} / \mathrm{sec}$ ) to achieve a desired compressional force ( 8 or, in most experiments, $20 \mathrm{gm} \mathrm{wt}$ ). This force was maintained in a static position for a specified time interval (2-6 sec), which was followed by withdrawal. During neurophysiological experiments, the step was displaced horizontally by $0.1,0.25$, or 0.5 $\mathrm{mm}$ when it was off the skin between trials (an interval of about $6 \mathrm{sec}$ ) so that, by the end of the run, the step had been applied to a succession of horizontal positions across a fiber's receptive field. An experimental run started with the contact oval being under the higher, flat side of the step and ended with it being entirely on the lower, flat side. During psychophysical experiments, the step was indented at a single central locus on the fingerpad. The compressional force of the plate against the skin, the displacements, and the velocities of the plate were controlled with local analog feedback circuits driven by a minicomputer. The reactive force of the plate against the skin was measured with a force transducer in contact with the top of the spring-loaded plate containing the step. The sequence of stimuli delivered and stimulus timing were also controlled by the minicomputer.

All stimuli were delivered to the volar surface of the distal phalanx of the finger. The hand was restrained in plasticine, with the finger to be stimulated held at a slightly higher level. In neurophysiological studies, this finger was restrained by a plastic screw glued to the fingernail 
and sunk into the plasticine. The sides of the fingerpad were not restrained, thereby allowing the fingerpad to deform as it might if the fingertip were actively pressed against a passive surface.

Neurophysiological experiments. Action potentials were recorded from single mechanoreceptive afferent fibers in the upper or lower median and ulnar nerves of 8 anesthetized juvenile Macacca fascicularis monkeys weighing 4-6 kg. The methods of electrophysiological recording, fiber dissection, and fiber classification were similar to those previously described (Talbot et al., 1968). Only those fibers with receptive fields centered close to or on the center of the volar distal pad of the second, third, or fourth finger were used in this study. Mechanoreceptive afferents were classified as being slowly adapting (SA) if they exhibited a static discharge for more than 2 sec during a steady indentation of the skin produced by a $50 \mathrm{gm}$ wt resting freely on the skin. All were of the Type I, and not the Type II variety, as described for humans by Johansson and Vallbo (1979). Fibers responsive only to the dynamic phase of skin indentation were classified as "rapidly adapting," or RA (Meissner corpuscle type) or "Pacinian." RA and Pacinian fibers were distinguished on the basis of the sizes of their receptive fields and responsiveness to remote stimulation. Pacinians had poorly defined boundaries and responded to low-intensity taps to the dissection table or to vibratory stimulation of one or several digits and/or a large part of the palm. The RAs and SAs described in the present experiments were the same fibers studied in the 2 previous papers (LaMotte and Srinivasan, $1987 \mathrm{a}, \mathrm{b})$.

A dissection microscope equipped with a video camera allowed a $20 \times$ magnified view of the stimulated skin through the transparent plate as the plate was pressed onto the skin. The output of the camera was viewed on a video monitor and was recorded with a video cassette recorder. A video digital voltmeter placed between the camera and the video cassette recorder mixed the video input with (1) a numerically displayed voltage from the force transducer (updated every other video frame); (2) the cumulative number of action potentials from the start of a trial (updated each frame); (3) individual action potentials, each displayed as it occurred as a short horizontal line within a thin vertical strip at the left edge of the screen; (4) elapsed time in seconds from the start of the trial; and (5) the video field number, which continuously cycled from 0 to $59 / \mathrm{sec}$.

A PDP $11 / 34$ computer collected analog signals at sampling intervals of $20 \mathrm{msec}$ from transducers that indicated the vertical displacement of the stimulus plate and the compressional force. The computer also collected digital information representing the time of occurrence of each action potential, each video field, and the start of each trial.

Prior to the machine-controlled tactile stimulation, the receptive field of each fiber was mapped using von Frey monofilaments, each of which delivered a force that ranged from $0.08 \mathrm{gm}$ wt $(0.127 \mathrm{~mm}$ diameter $)$ to $1.9 \mathrm{gm}$ wt $(0.29 \mathrm{~mm}$ diameter). The papillary ridge pattern was drawn on an acetate sheet placed over the video monitor. Pen marks were superimposed on the drawing of ridges to indicate the skin location of each von Frey filament when one or more nerve impulses were evoked. Lines were drawn around the areas within which the filaments evoked a response. After mapping the receptive field, a vertical line was drawn on the acetate sheet at a location approximately parallel to the ridges and near the edge of the receptive field obtained with the stiffer von Frey filament. On the back of each plate (the side not coming into contact with the finger) was a thin black line that marked the start of the curvature from the high to the low side of the step (the dashed vertical line on the inset, Fig. 4). This reference mark was lined up with the line drawn on the acetate sheet by manual adjustment of the tactile stimulator. The sequence of vertical indentations was applied after ensuring that the entire contact oval would be on the higher, flat side of the step during the first few indentations.

Psychophysical experiments on human subjects. The sensory capacities of 5 naive humans to discriminate between different steps applied statically to the distal pad of the middle finger were measured. The finger was elevated slightly above the rest of the hand and restrained in plasticine. Each step was brought down vertically, at a velocity of 4 $\mathrm{mm} / \mathrm{sec}$, onto the finger, such that the center of the step was roughly at the center of the contact oval, until a force of $20 \mathrm{gm}$ wt was reached. This force was maintained for $2 \mathrm{sec}$, after which the step was withdrawn from the skin at $4 \mathrm{~mm} / \mathrm{sec}$. In addition, on 2 of the subjects, experiments with only steps 3 and 4 were performed, with the 2 -sec steady indentation eliminated, and only an indentation and a retraction ramp given, each at $4 \mathrm{~mm} / \mathrm{sec}$.

On each trial, 2 steps were presented in 2 possible sequences, $S_{1}$ and
$\mathrm{S}_{2}$; a "standard" was always presented first, followed by either the same standard $\left(S_{1}\right)$ or a "comparison" $\left(S_{2}\right)$. The subject was instructed to give either of the 2 possible responses: to state whether the second step was the standard $\left(R_{1}\right)$ or the comparison $\left(R_{2}\right)$. The 2 stimulus sequences were randomized and presented with equal probabilities 24 times each for a given pair of steps. The step pairs used were $(1,2),(2,3),(3,4)$, and $(4,5)$. For 3 subjects, the steeper step was given as the standard, whereas for the other 2 subjects, the more gradual step was the standard. A biasfree measure of discrimination sensitivity, $d^{\prime}$, was calculated from the pairs of conditional probabilities, $p\left(R_{1} \mid S_{1}\right)$ and $p\left(R_{1} \mid S_{2}\right)$, which represented the proportion of trials in which the subject stated that $S_{1}$ occurred when, in fact, $S_{1}$ and $S_{2}$, respectively, were delivered (Johnson, 1980). The psychometric function $d^{\prime}$ was then plotted as a function of the difference in width $(\Delta W)$ between each pair of steps tested.

Statistical analyses. The significance of the main effect of step shape on various measures of mean SA and RA responses was tested using repeated-measures analyses of variance. The Newman-Keuls procedure (Winer, 1971) was used for pairwise comparisons between individual means. The significance level was set at 0.05 .

\section{Results}

Individual responses of $7 \mathrm{SA}$ and $9 \mathrm{RA}$ mechanoreceptive fibers in the monkey's fingerpad were obtained. The responses of the SAs will be described first, followed by a description of the RA responses.

In order to see the deformation of the skin surface under a step, videotape recordings of the indented skin, illuminated from behind, were obtained. The skin surface profiles under steps 1 , 3 , and 5 were similar to the corresponding profiles during stroking (see Fig. 4, LaMotte and Srinivasan, 1987a). During the sequence of vertical indentations by a step, as described in Materials and Methods, a point in the contact oval first came into contact with the high, flat side of the step. After several indentations, the top, curved portion of the step came into contact with the point, which was followed by a "gap" or a loss of contact with the step for steep, but not for gradual, steps. Subsequently, after a few indentations, the point on the skin regained contact with the low, flat side of the step. Thus, under successive indentations by a step, the sequence of shapes assumed by the indented skin at any spot in the contact oval was flat, concave, and convex, followed by the flat shape again.

\section{Slowly adapting afferent fibers}

Responses to a single step. Shown in Figure 1 are the responses of a typical SA (S21) to a succession of indentations (by step 1, width $=0.45 \mathrm{~mm}$ ) across the receptive field along an axis perpendicular to the long axis of the finger. Also shown are the receptive fields mapped by using von Frey monofilaments and the actual force trace (typical) obtained during one of the trials. The temporal sequence of action potentials evoked during each trial is indicated on a horizontal line. This line identifies the position on the receptive field of the reference mark on the step, which was located at the beginning of curvature on the high side of the step. In order to have a good measure of the response to the sharpest portion of each of the steps, indentations were closely spaced when the reference mark was in the neighborhood of the most sensitive spot in the receptive field.

In the first few indentations, the entire contact oval was on the flat, high side of the step. The slight reduction in response to these successive indentations was due to intertrial "habituation" of the SA, perhaps owing to fatigue of the SA or incomplete skin recovery (Pubols, 1982b). However, the response to the flat portions on both the high and the low sides of the step was almost constant. As the sharpest portion of the step 


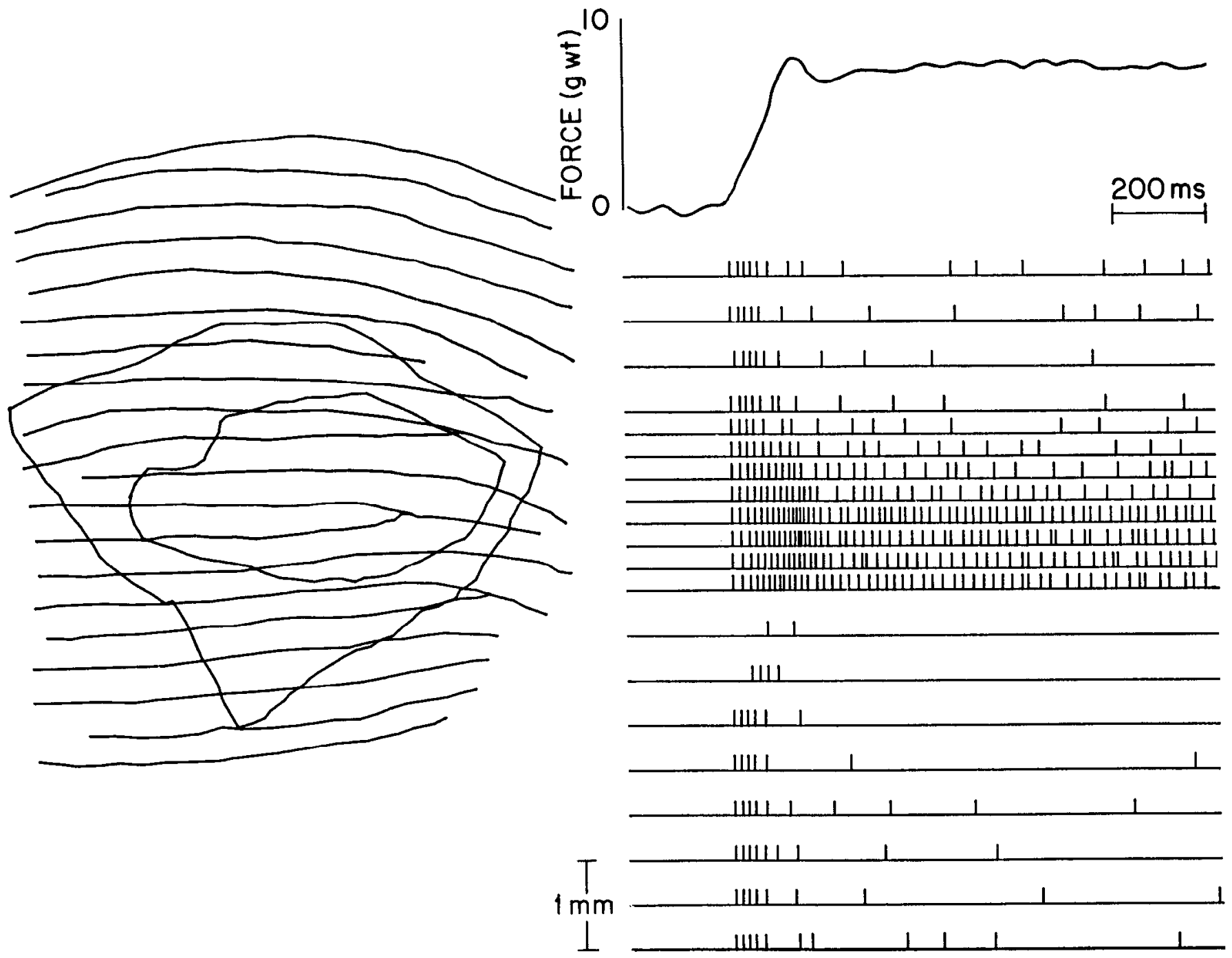

Figure 1. Responses of an SA (S21) to a succession of indentations across the receptive field by step $1(W=0.45 \mathrm{~mm})$. The velocity of indentation was maintained at $4 \mathrm{~mm} / \mathrm{sec}$ until the desired force $(8 \mathrm{gm} \mathrm{wt})$ was achieved, after which the force was maintained constant. On top is shown the actual force trace obtained (sampled at $20 \mathrm{msec}$ intervals) during one of the trials. The oval-shaped receptive fields obtained using von Frey monofilaments $(0.57 \mathrm{gm}$ wt for the inner area and $1.35 \mathrm{gm}$ wt for the outer) are shown on the left, superimposed on a drawing of the papillary ridges. The temporal sequence of action potentials obtained during each trial is indicated on a horizontal line identifying the position (on the receptive field) of the reference mark on the step, which, in turn, was located above the sharpest part of the step. The step indentations were shifted along an axis perpendicular to the papillary ridges. The progression of the trials was from top to bottom and the spatial interval between 2 consecutive trials was either 0.5 or $0.25 \mathrm{~mm}$, depending on the proximity of the reference mark on the step to the most sensitive spot in the receptive field. Left and right sides of the figure are drawn to the same scale.

approached the most sensitive spot, the discharge intensity increased to a peak response, followed by a sudden change to an almost silent response that was characterized by an increased latency in evoking the first impulse, very few impulses during the ramp indentation, and the absence of a steady response. For the steep steps, this corresponded to a gap that developed between the skin around the most sensitive spot and the steps. For the gradual ones, although no visible gap developed, the response was considerably reduced. As the low, flat side of the step increasingly occupied the contact oval, the responses again approached the earlier basal value of discharge. All the SAs exhibited the features mentioned, with variations in the intensity of basal and peak responses. The location of the peak response roughly corresponded to the center of the inner von Frey receptive field. It should be noted that because the total force on the finger was controlled, there was no difference in the applied stimulus, regardless of whether the contact oval was entirely on the higher or the lower flat side of the step.

It is instructive to interpret the results shown in Figure 1 as a population response. Thus, if the step indented the skin that contained identical SAs under each of the horizontal lines, then the population response would be approximated by the responses shown. It is then clear that the SAs under the sharpest portion of the step would respond most vigorously, that those under the "gap" would respond least vigorously, and that those under the flat parts of the step would provide a basal response between the 2 extremes. The fact that the skin is concave under the sharpest part of the step, convex under the gap, and flat elsewhere supports the hypothesis that SAs are sensitive to changes in the curvature of the skin, which, in turn, are directly related to the shape of the indenting object.

In order to show the progression of the population response 


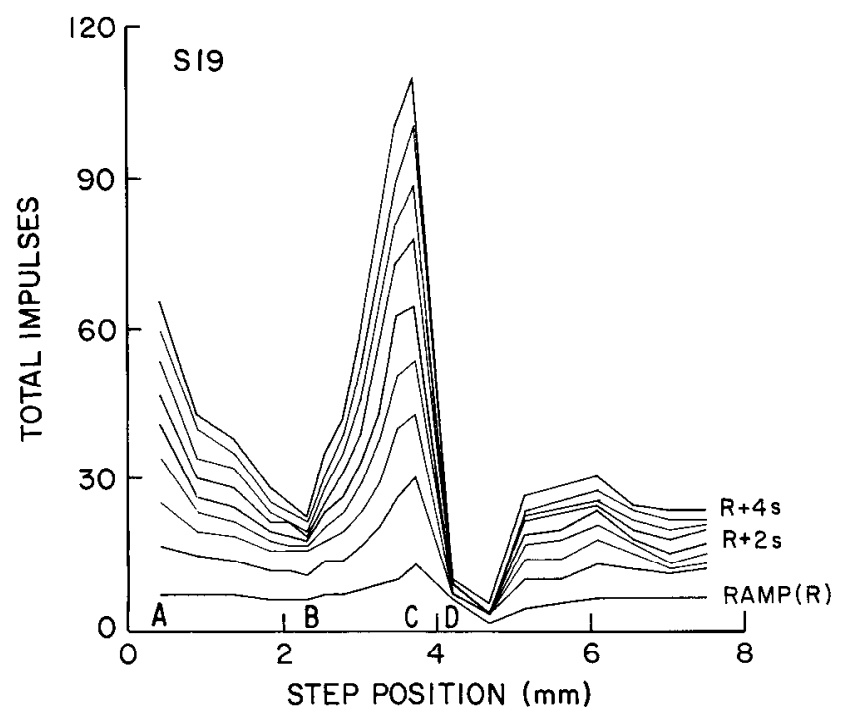

Figure 2. Spatial response profiles of a typical SA (S19) constructed from responses to successive indentations across the receptive field by step 1. Lowest curve indicates the total number of impulses during the indentation $\operatorname{ramp}(R)$. Subsequent response profiles were obtained at $0.5 \mathrm{sec}$ time intervals after the end of the ramp and represent the cumulative number of impulses at the respective times.

with time, the spatial response profiles at the end of the ramp phase of indentation and at $0.5 \mathrm{sec}$ time intervals thereafter are plotted for another typical SA in Figure 2. At the end of the ramp, the major features of the response are as described above. That is, the peak response occurred under the maximum change in curvature of the skin, the minimum response under the minimal change in curvature (i.e., under the gap), and a basal response under the flat parts of the step. As time progressed and the indentation was held steady-except for some distortion caused in the shape of the response profiles on the first few trials due to intertrial habituation of the SA-the same major features of the profiles were preserved to a remarkable degree. The effect of such preservation over time was to increase the contrast between the maximum and minimum response, thereby providing an "amplified" representation of the step in the neural response.

Clearly, the changes in the response profiles over time are a direct consequence of the adaptation behavior within each trial at various positions of the step. Shown in Figure 3 are the discharge rates as functions of time for 4 positions of the step. The frequency of discharge during the ramp is much higher than the adapted steady frequency in a single trial, and the higher the ramp frequency, the higher the steady value it settles at. This latter property is responsible for the preservation of the shape of the spatial response profile over time and the consequent increase in contrast between the maximum and minimum responses. Thus, SAs are sensitive to the change in curvature of the skin during the ramp, as well as during the stcady phascs of indentation. It is also interesting to note, from a modeling viewpoint, that the transition time from the end of the ramp to the time when the steady discharge rate is obtained is almost constant for all the trials and, therefore, is independent of the magnitude of response.

The effect of changes in the step shape on SA responses. We next present the responses to controlled changes in shape resulting from varying the half-cycle wavelength of the sinusoidal

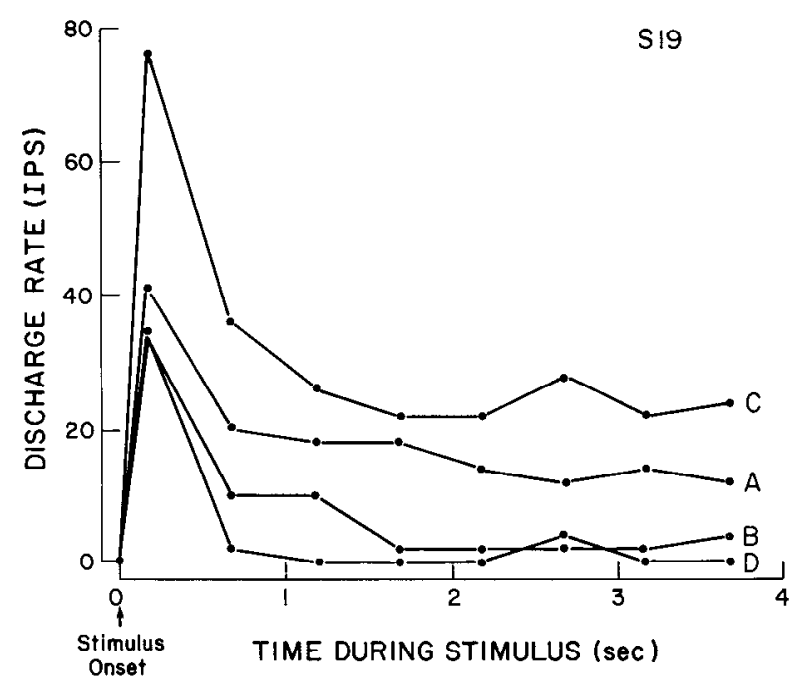

Figure 3. Discharge rate (impulses/sec) of an SA (S19) as a function of time for a few selected trials. Data were obtained from Figure 2 . In each trial, the discharge rate during the ramp, as well as at $0.5 \mathrm{sec}$ intervals thereafter, was calculated and linearly interpolated. Trials $A-$ $D$ correspond to the first, sixth, twelfth, and thirteenth trials (as indicated in Fig. 2). Notice the drastic change in response to 2 neighboring indentations, $C$ and $D$, spaced $0.5 \mathrm{~mm}$ apart.

steps while keeping the height constant. Such changes, in effect, vary the curvature in various portions of the step, as well as the step width. The 6 steps used are as shown in the inset in Figure 4 , and could be broadly classified into 2 groups, namely "steep" (0-3) and "gradual" (4 and 5).

The spatial response profiles of $S 21$ for each of the steps at 1 sec after the ramp are shown in Figure 4. Since the reference mark on the step was just adjacent to the sharpest point, the particular process by which each of the profiles was aligned (see legend, Fig. 4) resulted in occurrence of the peak response of each profile at the same step position on the skin. On the other hand, the minimum response locations for steep and gradual steps were distinctly different, owing to the considerable difference in the wavelengths of the 2 groups. However, even though step 5 was approximately 7 times wider than step 1 , the difference in the widths of the "modulatory region," defined as the distance between the beginning of the rise to the peak response, and the minimum response was not as drastic. Obviously, the discharge profile width of an SA is not directly related to the wavelength of a step, but to the skin surface profile underneath it. When the skin profiles under static indentation by the steps were observed, using a fiber-optic light placed behind the steps, the profiles were found to be similar to those under stroking (see Fig. 4, LaMotte and Srinivasan, 1987a). It was indeed confirmed that the changes in wavelengths of the skin profiles under the steep and gradual steps corresponded more to the changes in the width of the modulatory region in Figure 4 than to the changes in the actual wavclengths of the stcps. For example, the wavelength of the skin profile under step 5 was about 1.5 times that under step 1, and was thus approximately equal to the ratio of the widths of the modulatory regions in Figure 4 rather than to the ratio of the wavelengths of these steps, which was 7 . Within the steep or gradual groups of steps, there was only a small difference in the skin surface profiles, which, in turn, is reflected as small changes in the width of the modulatory region.

The magnitude of the peak response diminished as the step 


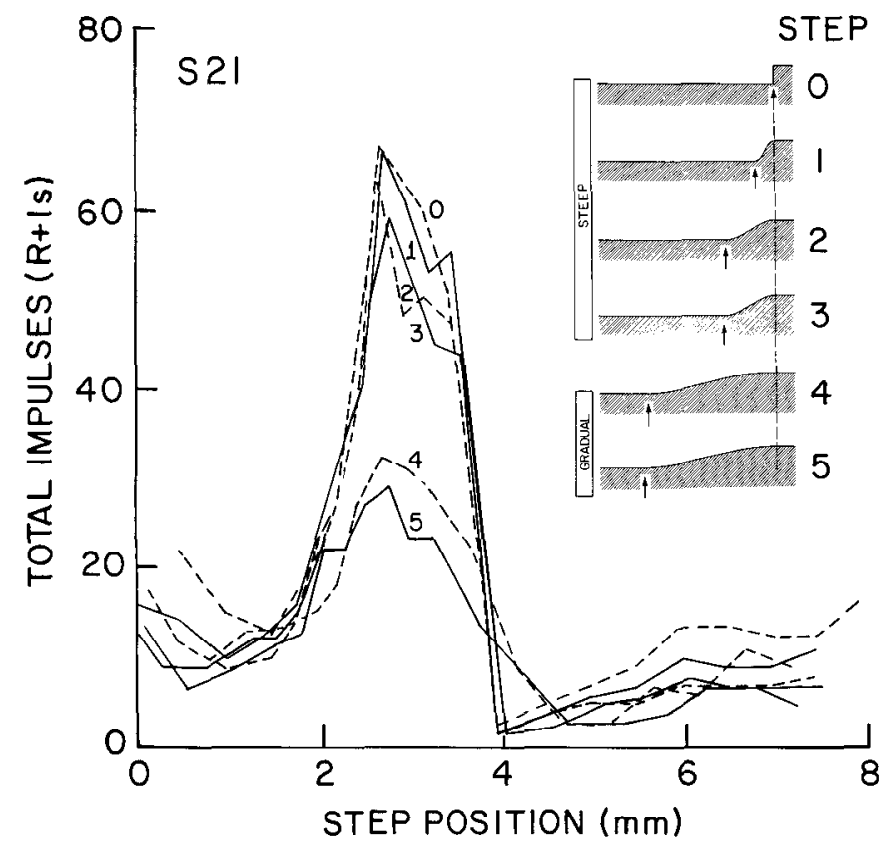

Figure 4. Spatial response profiles of an SA (S21) to steps of different shapes. The cross-sectional profile of each step is shown in the inset. The cumulative number of impulses at $1 \mathrm{sec}$ after the end of the ramp is plotted for each of a succession of step positions of each step $(0-5)$ on the skin. The responses to steps 0,2 , and 4 are indicated by dashed lines. These profiles were aligned so that, for a given step position, the reference mark on each step was at the same spot on the skin. Since the reference mark on each step was directly above the sharpest portion of the step, the location of the peak response coincided for all the steps. However, the locations of minimum responses for steep and gradual steps did not coincide, owing to the large difference in the wavelengths of the steps, which caused the width of the skin profiles to differ significantly. In the inset (not the same scale as the plot), the arrows and vertical line indicate, respectively, the beginning and end of the sinusoidal portion of each step.

curvature became more gradual and, at least for sensitive SAs, the reductions were clearly noticeable even between steps within the groups of steep and gradual. Since the curvature of the top portion of the step decreased as step number increased (with an especially large change between steps 3 and 4), and the peak responses reflected these changes remarkably well, the hypothesis that $\mathrm{SA}$ responses are directly related to changes in curvature at the most sensitive spot is further strengthened. It should be noted that, at the sharpest part of each step, the skin would closely follow the shape of the step and, hence, there would be a significant difference in the skin surface profile at that spot between any 2 steps used.

Even though the SA whose responses are shown in Figure 4 seems to discriminate the steps in terms of the peak number of impulses, it can be seen that the basal response to various steps varies (probably because of varying degrees of habituation at the time of application of each step) at least as much as the difference in peak response for steps within the groups of steep and gradual steps. Thus, the significance of the peak response alone is suspect, and the difference between the peak and the basal response (taken just prior to onset of the rise to the peak, as estimated by eye) for each step is a better measure of the number of impulses elicited by that step. In Figure 5, for the times shown, the base response was subtracted from the peak response, and the result plotted as a function of step width, with

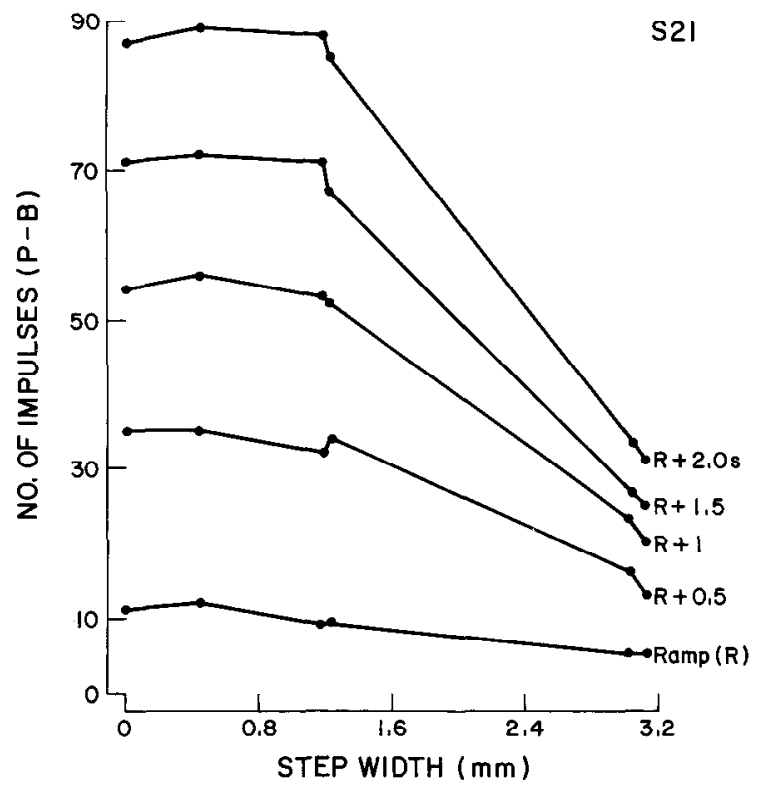

Figure 5. Effect of step shape on the peak response (above base) of an SA (S21) obtained from the spatial response profile generated by each step at selected times. The basal number of impulses was subtracted from the peak number of impulses $(P-B)$ (e.g., Fig. 2) and plotted as a function of step width. The basal response was defined as the number of impulses occurring just before the rapid rise to the peak. The lowest trace represents the impulse count at the end of the ramp, and the remaining traces were determined at successive time intervals of 0.5 sec.

time as a parameter. While the discrimination between steps 0 3 is not as good as the peak response alone would lead us to expect, it can be seen that the difference between the responses to steep and gradual steps keeps increasing as time progresses. This, again, is a consequence of the sensitivity of SAs to curvature change: The higher the change in curvature, the greater the magnitude of response during both the ramp and steady phases of indentation. Even though steps 2 and 3, as well as 4 and 5 , are very close to each other in shape, the SA responses to these pairs are not as close as would be expected, resulting in the abrupt changes apparent in Figure 5. The predominant reason for such changes is probably the difference in the level of habituation of the fiber at the time of presenting either one of the steps as a stimulus.

Individual differences in $S A$ response. Our interpretation of the response of a single SA under various positions of a step (see Fig. 1) as a population response is possible only if we assume identical characteristics for the skin and the SAs located at those positions. A more realistic population response, however, is much more complex, owing to the variability in the mechanical properties of the skin, the location and orientation of the mechanoreceptive fiber terminals, as well as their response properties, such as thresholds and sensitivities to indentation depth, velocity, and curvature. Thus, the SA population response necessarily has more jitter than the response of a typical SA, and the central nervous system has to extract the major features through appropriate processing.

Figure 6 shows the spatial response profiles obtained $1 \mathrm{sec}$ after the end of the ramp for steps 1 and 5 for 6 SAs plotted to the same scale. While the basal response is approximately the same for 4 SAs, it is lower for S13 and more than 3 times higher for $\mathrm{S} 25$. It is apparent that each of the SAs exhibited a narrower 

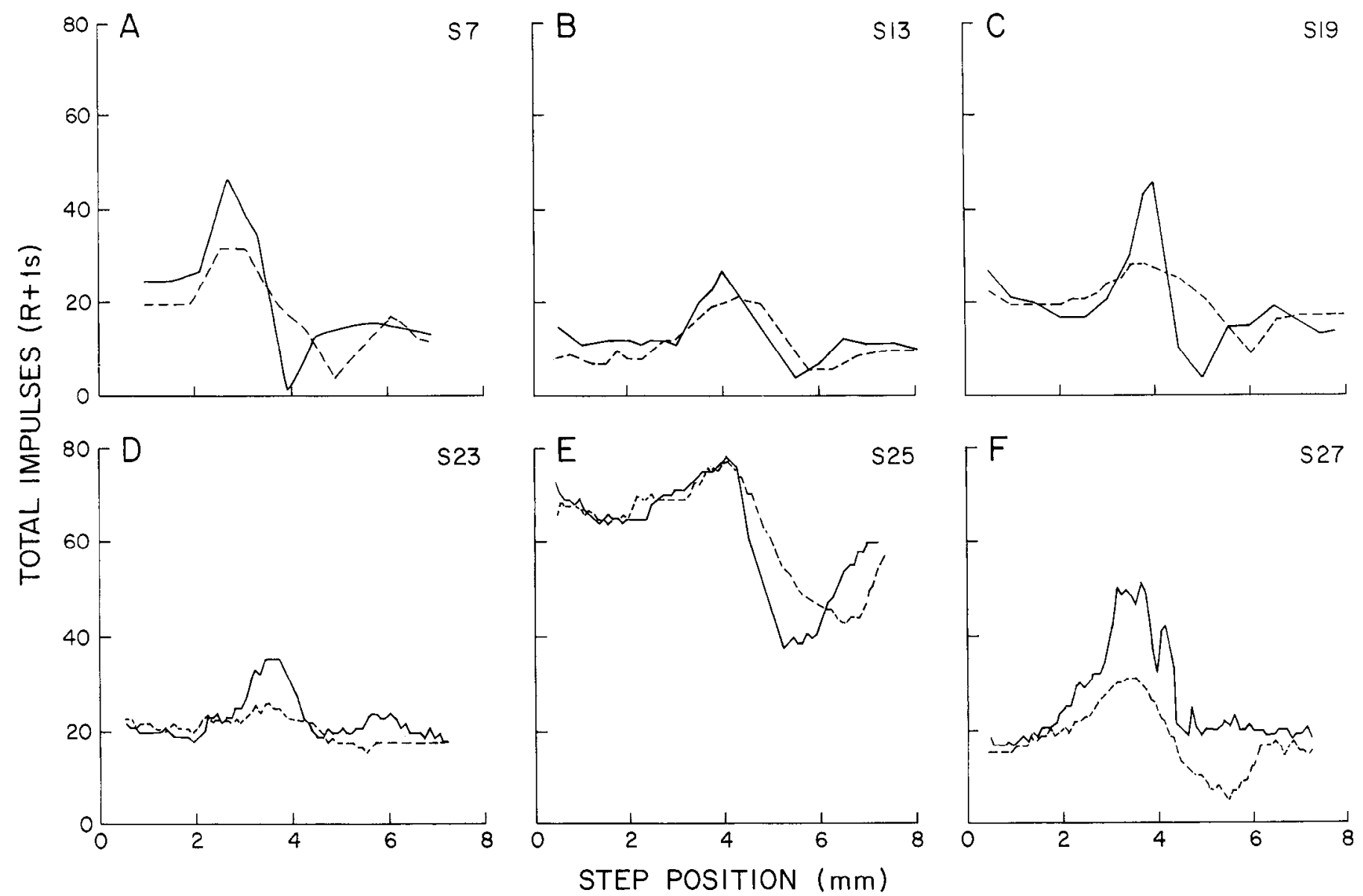

Figure 6. Individual differences in the responses of SAs to steps 1 and 5. Spatial response profiles at 1 sec after the end of the ramp are plotted. Solid and dashed traces represent the responses to steps 1 and 5 , respectively. The 2 traces were aligned to a single spot on the skin, as described for Figure 4. The indentations were spaced at 0.25 to $0.5 \mathrm{~mm}$ for the top 3 fibers, and at $0.1 \mathrm{~mm}$ for the bottom 3 . While the magnitude of the basal response varies from fiber to fiber, there is a clear modulation of the response when the step is in the vicinity of the most sensitive spot. The 2 steps are distinguished both by the magnitude of peak response and the width of the modulatory region, defined as the distance between the beginning of rise to the peak and the point of minimum response.

modulatory region and a higher peak response for step 1 than for step 5.

Mean SA response. Measurements of the major features of SA responses were obtained from the spatial response profiles for each step and averaged over all SAs. The measures of response widths were obtained by first fitting by eye a horizontal line through the basal response in a spatial response profile. In cases where the left and right basal responses differed, 2 separate horizontal lines were drawn. Then the distance between the points where the profile intersected with the horizontal line, from the rise to the peak to the fall from the peak, determined the peak width $P$; from the fall to the resumption of the basal response was defined as the gap width $G$. The combined peak and gap widths were denoted as $(P+G)$. The width from the
Figure 7. Effects of changes in step shape on spatial and intensive features of SA response. Response measures at $1 \mathrm{sec}$ after the end of the ramp, averaged over all the 6 SAs studied, are plotted as functions of step width. $A$, Measures of spatial features (mean widths): gap $(G)$, peak $(P)$, modulatory region $(M R)$, as well as the sum of peak and gap widths $(P+G)$ (see text). $B$, Measures of intensive features (mean response magnitude). Basal response subtracted from peak response $(P-B)$, and minimum response subtracted from peak response $(P-M)$.
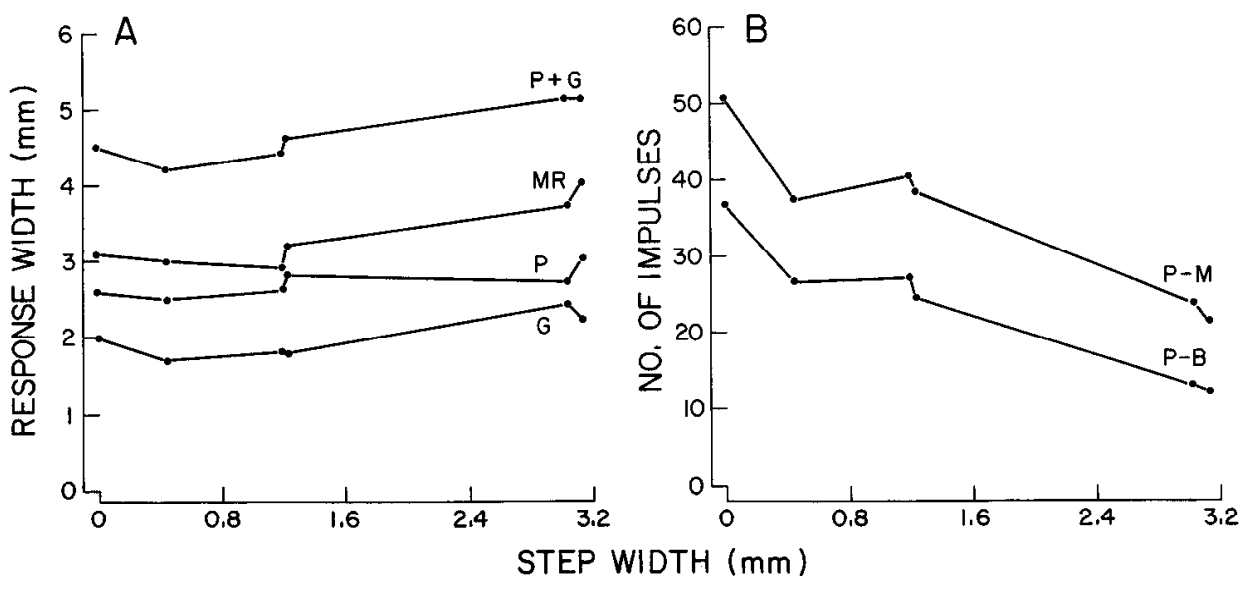

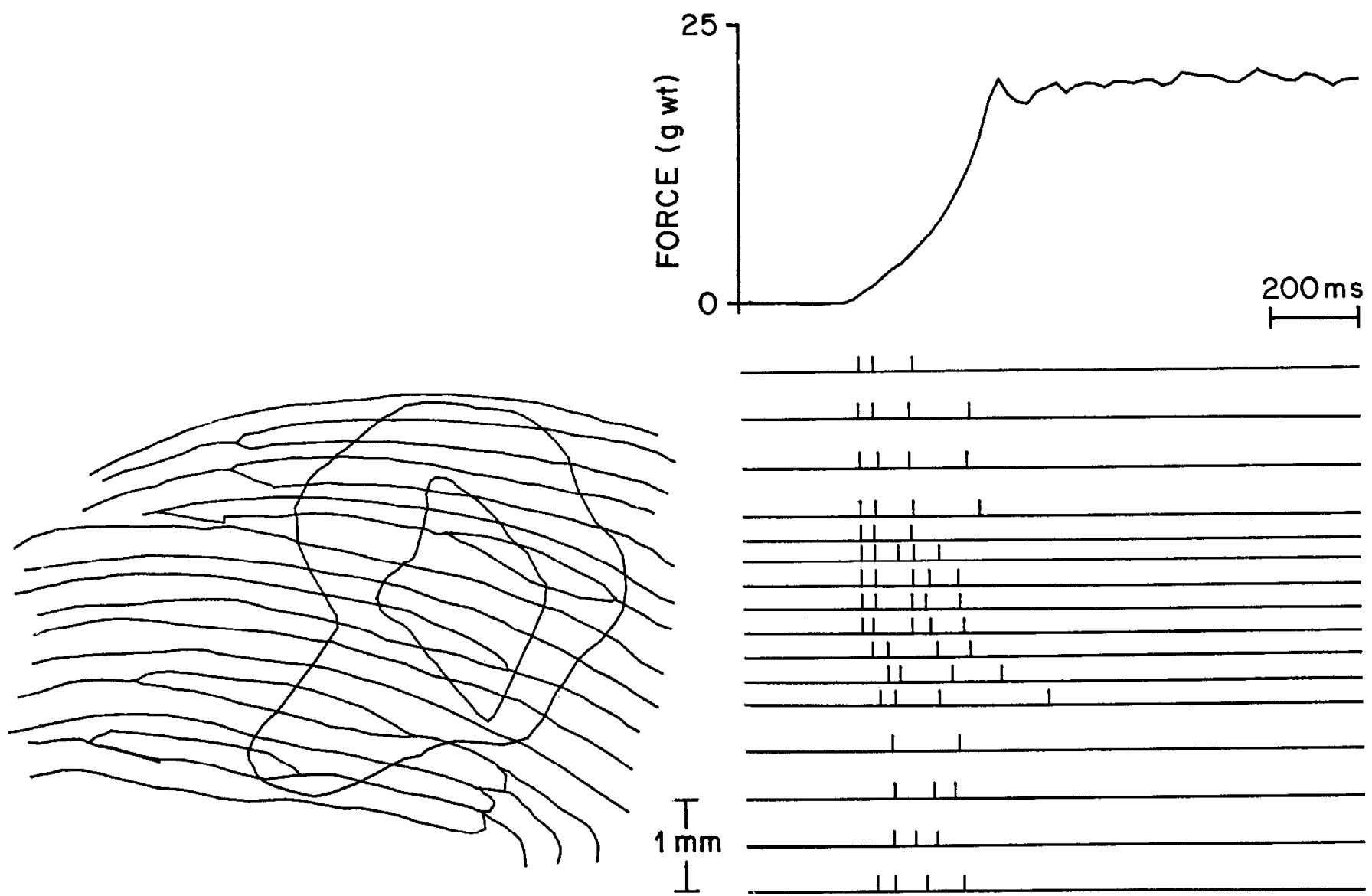

Figure 8. Responses of an RA (R25) to a succession of indentations across the receptive field with step $1(W=0.45 \mathrm{~mm})$. Same format as in Figure 1, except that a force of $20 \mathrm{gm}$ wt was used.

initial rise to the peak to the point of minimum response determined the modulatory region, $M R$. Measures of response magnitude were (1) the difference between the number of impulses at the peak and the minimum response, $(P-M)$, and (2) the difference between the number of impulses at the peak and the basal number of impulses, $(P-B)$. Each width and magnitude measure was averaged for all 6 SAs and plotted in Figure 7 as a function of step width. The measures of widths were defined as reflecting "spatial" features of SA responses to a step, while the measures of magnitudes represented "intensive" features.

Repeated-measures analyses of variance were performed on the above mcan measurements, followed by a pairwise comparison of individual means using the Newman-Keuls procedure. The level of significance was 0.05 . Since responses of only 3 SAs were obtained for step 0, the results shown in Figure 7 for this step were not included in the subsequent statistical analyses. While peak and gap widths did not vary significantly with different steps, the widths of the modulatory region and peakplus-gap widths were significantly different for the categories of steep (1-3) and gradual (4 and 5) steps. Within each of these groups, however, no statistically significant differences were found. The only significant differences in the magnitude measures $(P-M$ or $P-B)$ were also found between those for steep and gradual steps, but not for steps within either of these 2 categories. All the curves in Figure 7,A,B, displayed abrupt changes between 2 and 3 , as well as 4 and 5 , for the reasons given earlier (see Fig. 5).

\section{Rapidly adapting afferent fibers}

Responses to a single step. The responses of a typical RA to a succession of indentations across the receptive field by step 1 are shown in Figure 8, along with the von Frey receptive fields and a typical force trace obtained from onc of the indentations. Figure 8 is entirely analogous to Figure 1 which shows SA responses, and the same procedure and conventions were followed in obtaining it. Comparison of the 2 figures indicates that even during the indentation ramp alone, the RA responded with fewer impulses and at a lower rate than the SA; however, similarities such as the reduced response under the gap, accompanied by an increased latency in evoking the first impulse, are apparent for the RA. A few of the other RAs exhibited a slightly increased response as the sharpest portion of the step approached the most sensitive spot. However, such modulation was generally not the case for all the RAs, and only a few RAs seemed to be sensitive, to a limited extent, to the amount of or the rate of change in skin curvature.

Effect of changes in step shape on $R A$ responses. The spatial response profile for each of the steps obtained from a curvaturesensitive RA (R30) at $1 \mathrm{sec}$ after the end of the ramp is shown in Figure 9. This RA exhibited perhaps the best curvature sensitivity of all the RAs studied. For comparison with SA responses (Fig. 4), the spatial response profiles were plotted at 1 sec after the end of the ramp. However, except for a maximum of 3 impulses in the peak regions, most impulses were emitted during the ramp phase of indentation. The successive inden- 


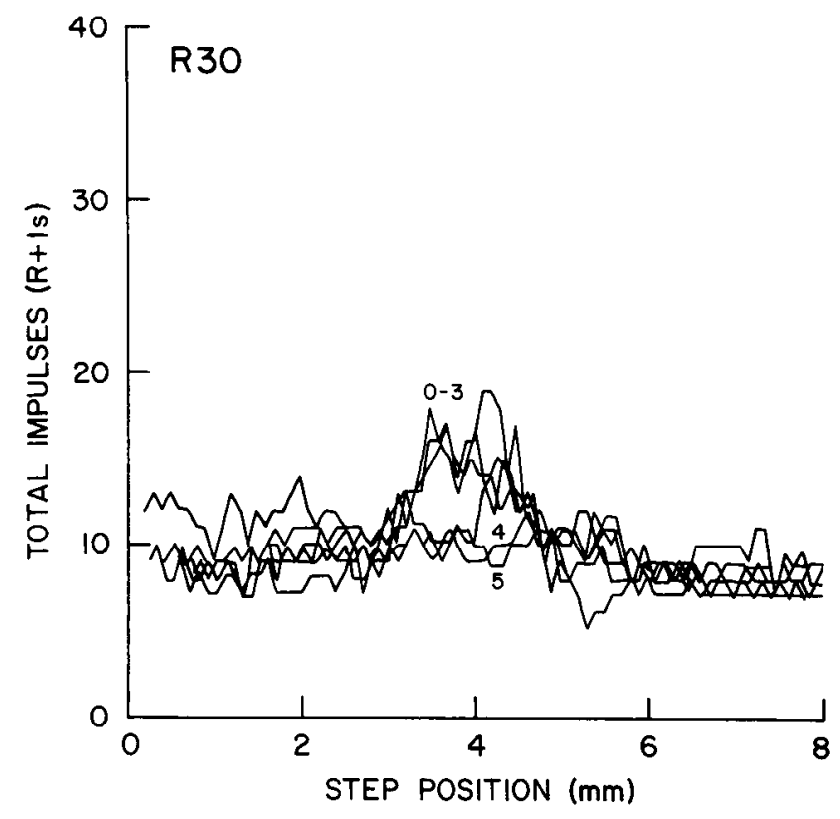

Figure 9. Spatial response profiles of an RA (R30) to steps of different shapes. Same format as Figure 4. Step numbers corresponding to each of the profiles are indicated on the respective curves. Except for a maximum of 3 impulses in the peak responses, the rest were emitted during the ramp phase of indentation. The profiles appear jagged because of differences of mostly \pm 1 impulse in the responses for successive indentations, which were closely spaced $(0.1 \mathrm{~mm})$. A comparison of these curves with those in Figure 4 shows a definite superiority of the SA response for discrimination of steps, both in the number of impulses and in the width of the modulatory region.

tations for each of the steps were closely spaced at intervals of $0.1 \mathrm{~mm}$ so as to obtain accurate measures of spatial features, such as the width of the modulatory region. This resulted in a jagged profile due to a difference of \pm 1 impulse in the response for successive positions, even under the flat portions of the steps. While the basal response for all the steps was approximately the same, the peak responses to the steep steps were considerably higher than those for the gradual steps and, hence, this RA could be said to have some curvature sensitivity. However, compared to the SA's response in Figure 4, the RA's response had fewer impulses and was much poorcr in providing any spatial information-for example, via the width of the modulatory region.

Individual differences in $R A$ response. In order to show the variability in the responses of different RAs, all 9 RAs' spatial response profiles to steps 1 and 5 are shown in Figure 10. Since a few of the RAs emitted 1 to 3 impulses slightly beyond the end of the ramp, the spatial response profiles are plotted at 1 sec after the end of the ramp. The number of impulses in the top 3 panels has been plotted at the same scale used for the SAs in Figure 6, to facilitate comparison. The other panels have been plotted at an enlarged vertical scale in order to enhance the weak modulation of responses under the steps.

In comparison with other RAs, the top 3 had a higher number of impulses during the basal response than did the RAs below. Even so, the greater discharge of those 3 RAs was much less than that of most SAs. For one RA, R28, the steps were vibrated (10 Hz, $50 \mu \mathrm{m}$ peak-to-peak) during the stcady part of indentation following the ramp. This increased the overall number of impulses, but did not improve the spatial modulation of the fiber's response to the steps. As is shown in Figure $10 C$, the magnitude of responses was twice that of R30 and R33, which did not receive vibration. Only $\mathrm{R} 30$ and $\mathrm{R} 28$ seemed to discriminate between steps 1 and 5 in terms of peak response. R18 responded more vigorously to step 5 than to step 1 , perhaps because of variable levels of habituation at the beginning of the set of indentations for each step. While it is possible that the entire modulatory region was not covered with step 5 for R16 or with both steps for R20, no clear spatial cues for discrimination are apparent in the responses of most of the fibers. Only R30 and R33, and to some extent R16, exhibited slight differences in widths of the modulatory regions for steps 1 and 5 that might provide some weak spatial information.

In summary, the RAs responded with far fewer impulses than did the SAs during the ramp phase of indentation. While there was some modulation of RA response when the sinusoidal portion of a step was in the neighborhood of the most sensitive spot in the receptive field, any differences in the positions of the maximum and minimum number of impulses were neither as clear nor as consistent as those of the SAs. In fact, even the discrimination between steps 1 and 5 on the basis of either the number of impulses or the width of the modulation region was quite uncertain for most of the RA responses shown.

Mean $R A$ response. Since the spatial features of peak widths and modulatory regions were not discernible in the spatial response profiles of most RAs, only the intensive features were considered in the analyses of mean responses of all RAs. Two intensive measures of the mean RA response to different steps are plotted in Figure 11. These are the mean peak-minus-minimal response $(P-M)$ and the mean peak-minus-base response $(P-B)$ as defined for Figure $7 B$. These means were subjected to repeated-measures analyses of variance, with pairwise comparisons of individual means using the Newman-Keuls procedure. The responses to step 0 , shown in Figure 11, were not included in the statistical analyses, since only 4 RAs were tested under this step. Only the response measure $(P-M)$ for step 1 differed significantly from the responses to the other steps; otherwise, not even the differences between the categories of steep and gradual steps were significant for either response measure. As is evident from Figure 10, contributions to the mean by steps R30 and R33 were much higher than those of other RAs, and therefore these 2 fibers had a dominant influence on the 2 curves in Figure 11. Since only the ramp impulses were taken into account, the contribution of $\mathrm{R} 28$ was not as high. If the responses of R30 and R33 were not taken into account in the means, then the 2 curves became much flatter, though there was still some difference between the average response to the steep steps $(P-$ $B=1.3, P-M=2.33)$, and the gradual ones $(P-B=1.05$, $P-M=1.8$ ). Comparing Figure 11 with Figure $7 B$, it is clear that corresponding measures of SA responses yield much higher numbers of impulses (hence, are less sensitive to the presence or absence of a single impulse) and provide much better discrimination between the steps.

Comparison of sensory discrimination performance of humans with discriminative responses of monkey $S A$ s and $R A$ s

The results of psychophysical measurements of sensory discrimination $\left(d^{\prime}\right)$ between the steps and the relevant intensive and spatial features of SA and RA responses are summarized in Figurc 12. In Figure $12 A$, the mean $d^{\prime}$ for 5 human subjects indicates that discrimination was not possible between steps 2 and 3 or 4 and 5, but did occur between steps 1 and 2 (for 3 of the 5 subjects) and steps 3 and 4 (all 5 subjects). The fact that, on the average, the RAs responded with far fewer impulses than 

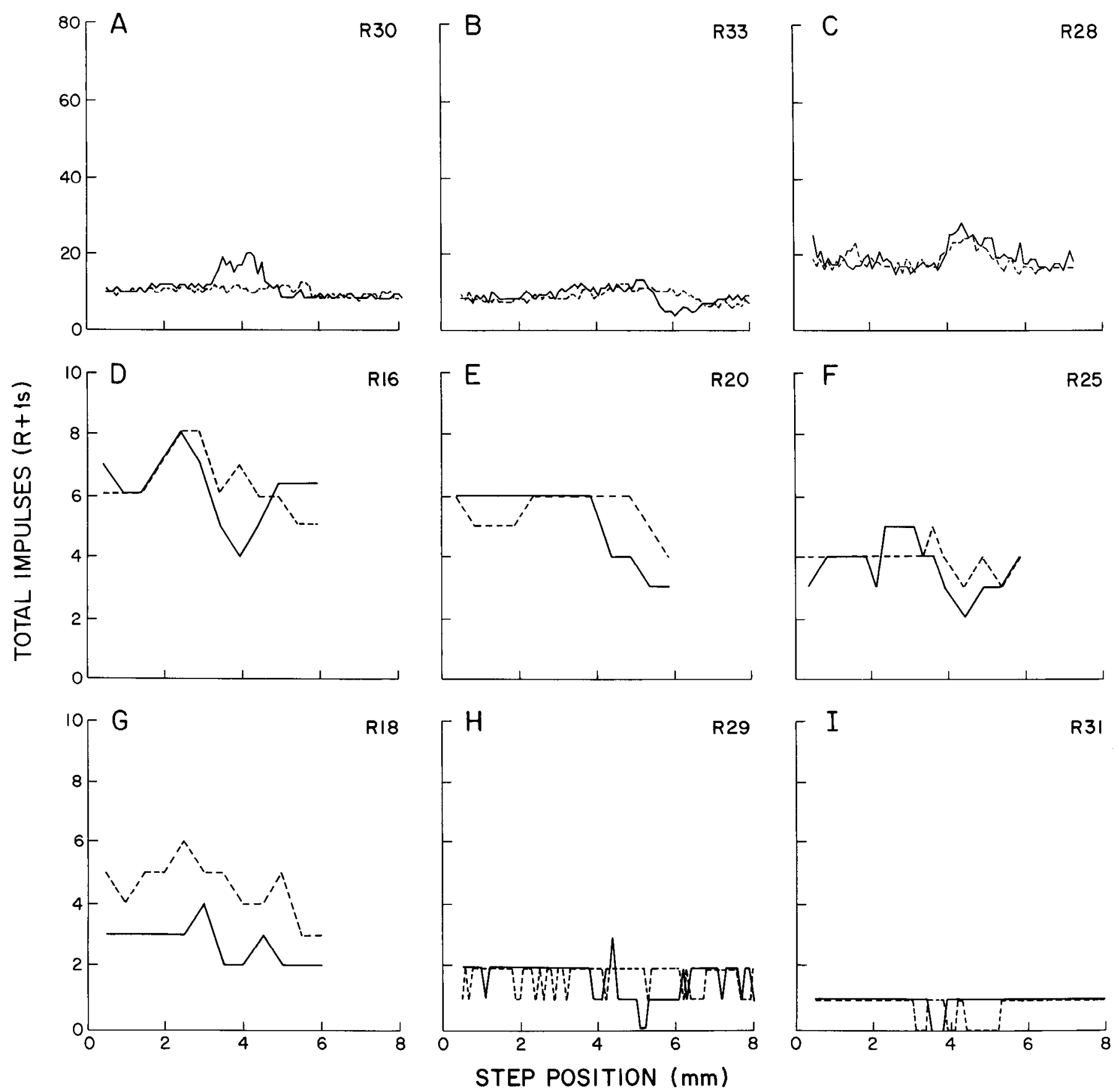

Figure 10. Individual differences in responses of RAs to steps 1 and 5. The solid and dashed traces represent the responses to steps 1 and 5 , respectively. The 2 traces were aligned with respect to each other, as explained in the legend for Figure 4 . Top 3 RAs responded more vigorously than the others and are plotted at the same scale as that of Figure 6 for SAs in order to facilitate comparison. The other RAs were plotted on an enlarged scale to emphasize the weak modulation of responses.

the SAs did is evident from Figure $12 B$; it also shows that both the intensive and spatial features in SA responses did almost equally well in discriminating the steps. Only the SA modulatory region for the step pair 3 and 4 was found to differ significantly from the other pairs. Since RAs responded with so few impulses, intensive cues from them were highly sensitive to their initial state and to the presence or absence of even one impulse, as indicated by the jagged curves in Figure 9. Therefore, their response, as a basis for discrimination, is suspect, as is supported by the flat curve in Figure 12B. We therefore conclude that SAs are primarily responsible for the discrimination between steps when the steps are vertically indented into the skin.

\section{Discussion}

It is known that when a flat object with sharp edges is applied to the skin statically or with superimposed vibrations, the SA responds much more vigorously when an edge is on its most sensitive spot than when the flat part occupies the contact oval (Vierck, 1979; Phillips and Johnson, 1981a; Johansson et al., 1982). Also, this edge sensitivity is much poorer, if not absent, 


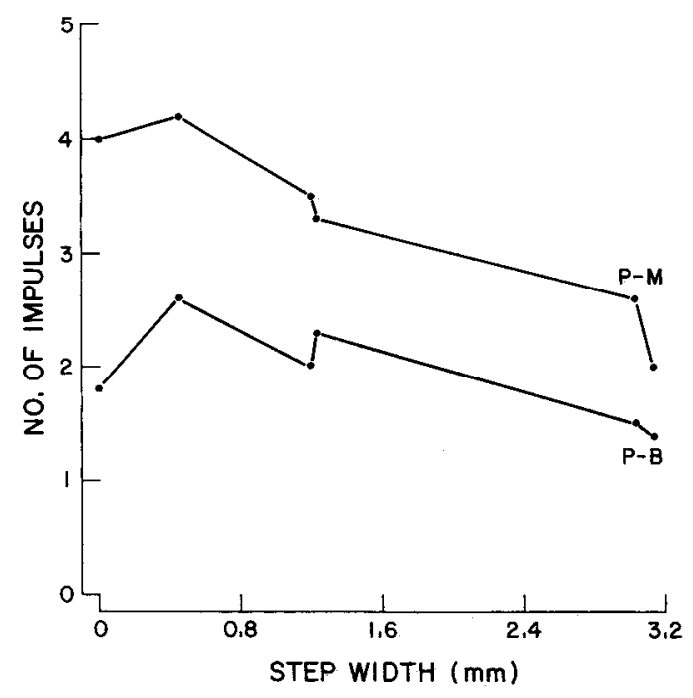

Figure 11. Effects of changes in step shape on intensive features of RA response. The mean number of impulses evoked during ramp by all 9 RAs was normalized by subtracting the basal response from the peak response $(P-B)$ and by subtracting the minimum response from the peak response $(P-M)$. The results are plotted as functions of step width.

in the responses of RAs or of Pacinian afferents. These receptor properties account for the general conclusion that when objects with spatially organized surface patterns, such as raised, braillesized dots and gratings, were applied statically or stroked across the skin, the spatial resolution of responses of SAs, and not of RAs or Pacinians, matched the tactile spatial resolution achieved by humans (Johnson and Lamb, 1981; Phillips and Johnson, 1981a).

In order to explain the edge enhancement in the steady phase of the SA response, a mechanistic model of the skin was proposed by Phillips and Johnson (1981b) and was used to calculate the stress and strain distributions within the skin when the gratings used in their experiments were applied to it. On the basis of the observation that the calculated compressive strain

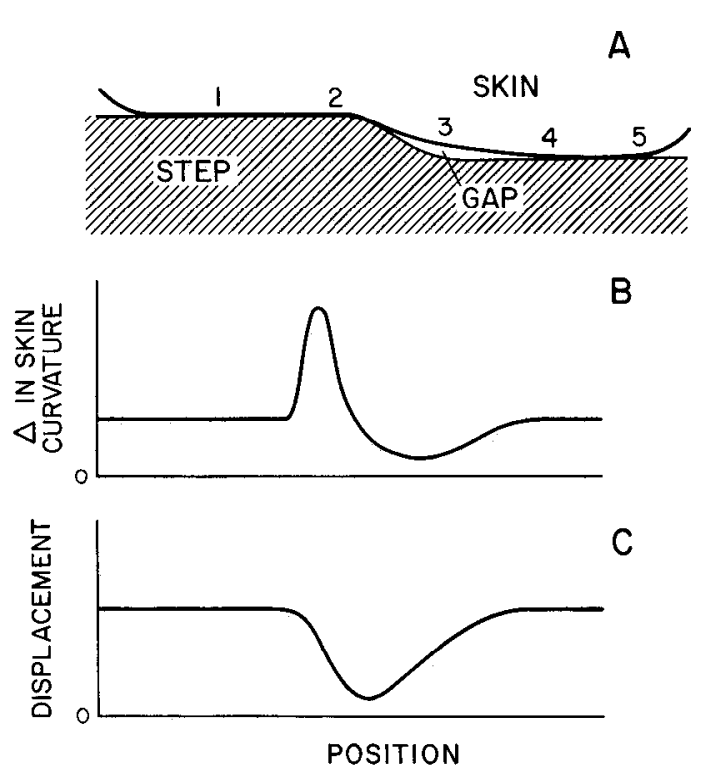

Figure 13. Model to interpret the observed responses of SAs and RAs. $A$, Schematic of the profile of skin deflection by the step (step 3). Numbers $1-5$ refer to certain equivalent positions of the most sensitive spot on the skin relative to the step, as the step indents the skin surface at successive lateral positions. $B$, Schematic of the sequence of changes in skin curvature of the most sensitive spot with respect to the resting state. The adopted sign convention is that concave skin curvature is positive. $C$, Schematic of the displacement of the most sensitive spot in the receptive field with respect to its resting state.

profiles under the gratings, at typical receptor depths, matched the corresponding SA static response profiles, they concluded that maximum compressive strain was the relevant stimulus for the SAs. In this model, 3 parameters - receptor depth, threshold, and sensitivity - were used to match the response profiles with the compressive strain profiles. The agreement was excellent, even though the parameters for a receptor were adjusted only once for all the gratings. However, neither the 3 model parameters nor the compressive strain at the receptor site are directly
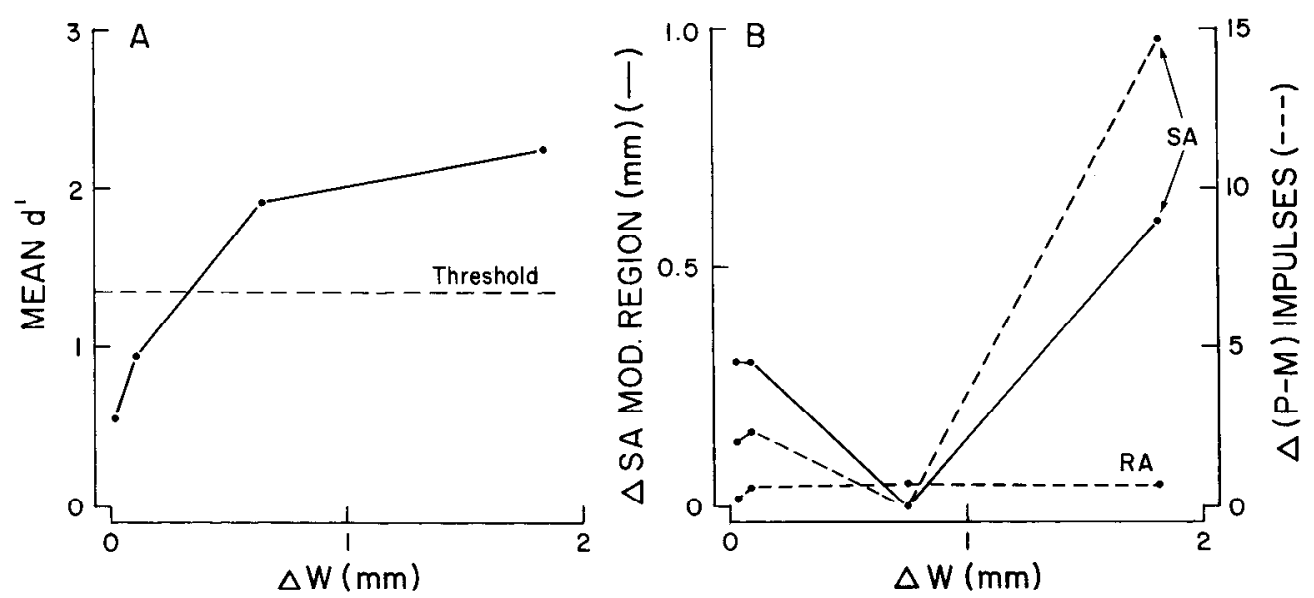

Figure 12. Discrimination of step shape. $A$, The mean, $d^{\prime}$, a measure of sensory discrimination, is plotted as a function of $\Delta W$, the differences in wavelengths between pairs of steps. The mean was obtained from data for 5 human subjects. Data points correspond, from left to right, to the step pairs $(2,3),(4,5),(1,2)$ and $(3,4)$, respectively. The value of $d^{\prime}=1.35$ was defined as the discrimination threshold. $B$, Difference in spatial and intensive measures of monkey SA or RA responses that might code for differences in step shape. The mean measures of response, in terms of the differences in response magnitude, i.e., the difference in the peak-minus-minimum number of impulses for each pair of steps, are plotted for RAs and SAs. Also plotted for SAs are differences in the width of the modulatory region. This was not plotted for RAs, since the modulatory region was not clear for most fibers. 
empirically verifiable at present. Therefore, we propose to interpret our results in terms of a model that relies on the events at the skin surface that can be directly observed.

It is known that for typical stimuli, SA and RA responses are predominantly influenced by the stimulus in the immediate neighborhood of the most sensitive spot in their receptive fields (Phillips and Johnson 1981a). All the results of previous experiments on SAs using probes that indented the most sensitive spot (e.g., Knibestöl, 1975; Pubols and Pubols, 1983) can be consistently interpreted as follows: The SA discharge ratc is a function of the displacement and velocity of the most sensitive spot, and generally increases with an increase in either variable. In addition, we hypothesize that the $\mathrm{SA}$ response also depends on the amount and rate of change in skin curvature at its most sensitive spot in the receptive field. Thus, edge sensitivity is only a special case of the general property of sensitivity to changes in skin surface curvature: When the edge is on the most sensitive spot, the change in curvature of the skin from its resting state is naturally much higher than the change in curvature when the flat part indents it, and this fact is directly reflected in its response. In addition, the observation of Phillips and Johnson (1981a) that the edge enhancement effect increased with an increase in the spacing of 2 neighboring bars can be explained as being due to the increasing local changes in curvature of the skin at the most sensitive spot because of the better conformation of the skin around the edge.

Consider also the indentation of the skin by a smooth object having no abrupt edges or corners. In such a case, the skin surface in the contact oval conforms to the object's surface and, thus, has the same local curvature. Therefore, if any mechanoreceptors are sensitive to the curvature of the skin at their most sensitive spots, then encoding of the local shape of the skin, which, in turn, is directly related to the local shape of the object, can be easily accomplished.

Having established the prima facie case for the curvature sensitivity of the SAs, we will now consistently interpret their responses to the smooth shapes used in our experiments. Indeed, in the 2 previous papers, which describe experiments in which the same steps were stroked across the skin under constant force, all the primary features of the data were consistently interpreted by hypothesizing that the SA response was highly sensitive to the amount and the rate of change in skin curvature at the most sensitive spot, while the vertical velocity of that spot predominantly influenced the RA response (LaMotte and Srinivasan, $1987 \mathrm{a}, \mathrm{b})$.

In analyzing the responses to vertical indentations, we will first describe the mechanical events on the skin, and afterward the responses. When the finger is not in contact with any object, the skin is at the resting state and the curvature at the most sensitive spot is therefore convex. Consider now that the step is stationary, and that when the finger is pressed on it (shown schematically in Fig. 13A), the contact area is big enough to cover all the positions on the step that are occupied by the most sensitive spot in the actual sequence of indentations. As the fingerpad comes in contact with a sequence of loci along the step, the local events at the most sensitive spot can be described equivalently if we visualize the spot itself as occupying successive positions from left to right on the skin surface (shown in Fig. 13A). Up to position 1 , the curvature of the indented skin at the most sensitive spot is 0 , representing a moderately positive change in curvature with respect to the resting state. In position 2 , the most sensitive spot is under the sharpest portion of the step, and, as shown, the skin curvature is concave, indicating a large positive change in curvature. In position 3 , a gap develops between the most sensitive spot and the step for steep steps, but not for gradual ones. In either case, the skin at the most sensitive spot has a convex curvature close to its initial state, and hence the change in curvature drops dramatically. Subsequently, in position 4 and beyond, because the total force of indentation is held constant, the deformation at the most sensitive spot is identical to that on the high, flat side (prior to position 1), i.e., the skin curvature at the most sensitive spot is 0 , therefore indicating a moderately positive change in curvature.

The corresponding changes in curvature (measured with respect to the resting state) for each of the positions of the finger on the step are shown schematically in Figure $13 B$. Comparing this with the response of an SA, S19, shown in Figure 2, we can see that, except for the effects of intertrial habituation on the left side of the figure (which can be eliminated by pre-adapting the fiber), each of the spatial response profiles imitates the curvature change profile of Figure $13 \mathrm{~B}$. As is apparent in Figure 3, this means that curvature sensitivity is present both during the ramp and steady phases of indentation. The spatial response profiles for all the steps shown in Figure 4 strongly confirm the curvature-sensitivity hypothesis: The peak number of impulses reduces monotonically as the steps become more gradual, and the maximum drop occurs between steps 3 and 4, where there is a drastic change in the curvaturc of the sharpest portion of the steps. Also, the minimum number of impulses for steep steps is 0 , while it is slightly higher for the gradual ones. This reflects the fact that a gap forms only for the steep steps and, therefore, the most sensitive spot is almost in the initial resting state, whereas, for the gradual steps, the most sensitive spot still has a small indentation, as well as a very small positive change in curvature. The shift to the right of the minimum response point for gradual steps is, as explained earlier, due to the change in skin profile under the steep and gradual steps. Skin profiles were observed in experiments where a fiber-optic light was placed behind the steps, and measurements indicated that the modulation region for gradual steps would be 1.2-1.5 times that for steep steps (confirmed in Fig. 4), even though the actual wavelengths of the gradual steps were 2.5-7 times those of the steep ones (not including step 0).

The responses of RAs to skin indentation by probes have been recorded previously for the glabrous skin (e.g., Knibestöl, 1973; Pubols and Pubols, 1983). These results can be interpreted by considering the RA discharge rate to be a function of the vertical velocity of the most sensitive spot in the receptive field. However, as Figure 9 indicates, a limited sensitivity to change in curvature or rate of curvature change is present, at least for some RAs. As shown in Figure 10, only half of the RAs tested showed any such sensitivity. The difference in the modulation region between steep and gradual steps was not clear in most cases. Thus, RAs do not appear to play a significant role in the perception of shapes of objects when the skin is vertically pressed down onto the object.

In order to explain consistently the limited modulation present in the RA response to steps, apart from curvature sensitivity, we take the same approach that was used to explain RA responses to the lateral stroking of steps (LaMotte and Srinivasan, 1987 b). It is known from vertical indentation experiments with blunt probes on RA receptive fields that the discharge rate is nonlinearly related to the velocity of indentation due to the presence of threshold and saturation phenomena. The discharge 
rate-velocity relationship is usually a monotonically increasing, sigmoidal-shaped curve (Knibestöl, 1973; Pubols, 1980). In the middle range of velocities, the relationship is approximately linear and, therefore, it is reasonable that a first analysis would neglect the nonlinear effects. Consider an RA whose discharge rate (number of impulses, divided by ramp time) is proportional to the vertical velocity (the depth of indentation at the end of the ramp, divided by the ramp time) of the most sensitive spot in its receptive field. Consequently, the number of impulses cmitted by the RA during the ramp is directly proportional to the depth of indentation at the most sensitive spot, irrespective of the ramp velocity. One implication is that the spatial response profile of this RA at the end of the ramp, obtained by sequential vertical indentation of an object with an arbitrary surface shape, would be proportional to the skin surface deflection profile under the same object. Indeed, this was found to be true for the spatial response profiles of RAs to the application of gratings (Phillips and Johnson, 1981a). Thus, in our present experiments, the spatial response profile of the RA that is not curvature-sensitive and whose discharge rate is linearly related to the velocity of the most sensitive spot would follow the skin displacement profile shown in Figure 13C. However, sensitivity to curvature change or rate of curvature change, the lack of linearity in the discharge rate-velocity relation due to threshold and saturation, as well as the presence or absence of an impulse owing to extraneous causes, act to distort this picture. It is seen that the addition of mild curvature sensitivity to a response profile similar to the displacement profile shown in Figure $13 C$ yields approximately the observed response profiles shown in Figures 9 and 10. While it is not possible to decide from indentation experiments alone whether this sensitivity is to the amount or to the rate of change in skin curvature, conclusions derived from RA response to stroking by steps (LaMotte and Srinivasan, 1987b) indicate that it should be the curvature rate sensitivity.

\section{Sensory discriminations of step shapes}

It was pointed out previously that the curvature sensitivity of SAs is present during both ramp and steady phases of indentation. Therefore, the much larger change in curvature of the skin under the sharpest portion of the step, as compared to that at the gap, causes an increased rate of discharge, resulting in an increase in spatial contrast in terms of the difference, with time, in the number of impulses between the peak and minimum responses in a spatial response profile. Another implication is that the difference in spatial contrast between 2 different steps also increases with time; i.e., spatial contrast increases more rapidly for steep steps than for gradual ones. Thus, the human capacity to discriminate between steps would be expected to improve with time. In psychophysical tests on 2 human subjects, we gave only ramp up and ramp down (no steady indentation); one subject could not discriminate even between steps 3 and 4 , while the other was able to discriminate, but did better with ramp plus $2 \mathrm{sec}$ than with ramp alone (unpublished observations). Since RAs become silent after the ramp phase, any such improvement in discrimination over time must be due solely to the SAs.

Psychophysical tests on humans demonstrate that discrimination between steps 3 and 4 is easily accomplished. Even steps 1 and 2 are discriminated between, but not as well. As supported by the SA response measures at $1 \mathrm{sec}$ after the end of the ramp, as shown in Figure 7, the neural cues for discrimination between steps 3 and 4 may be both spatial (e.g., width of modulatory region) and intensive (e.g., difference in the peak-minus-minimal number of impulses). The neural code for discrimination between steps 1 and 2, however, is probably only intensive, as is indicated for $\mathrm{S} 21$ in Figure 4. Even though some RAs appeared to provide intensive information for discrimination between steps 1 and 2, as well as both intensive and spatial information for discrimination between steps 3 and 4 (Fig. 10), none of these cues were as unequivocal as they were for SAs. Also, RAs are silent during the steady phase of indentation, even though thc sensory perception of shape remains. Hence, we conclude that, under vertical indentations, only SAs contribute to discriminations between differences in the shapes of the steps.

\section{Models of SAs and RAs for both stroking and vertical indentation stimuli}

In the present paper, the responses of SAs and RAs to vertical indentation by sinusoidal steps of varying shape were described. Two companion papers (LaMotte and Srinivasan, 1987a, b) have described the responses to stroking with the sinusoidal steps where stroke direction, step shape, and stroke velocity were varied. It was demonstrated that all the major results obtained with both stroking and vertical indentation stimuli could be explained consistently by the following hypotheses:

1. SA. The discharge rate of an SA is a function of the vertical displacement, vertical velocity, and the amount and rate of change in curvature of the skin at the most sensitive spot in its receptive field. The higher the value of any of these 4 quantities, the higher the discharge rate; however, when the rate of change of curvature is negative, the SA becomes silent.

2. $R A$. The discharge rate of an RA is a function of the vertical velocity and the rate of curvature change at the most sensitive spot in the receptive field. The higher the magnitude of either of these, the higher the discharge rate.

Here both the displacement and change in curvature (approximated by the second spatial derivative of displacement) are measured with respect to the resting state of the skin. Indentation displacements and curvature changes from convex towards concave are considered positive.

A goal of these hypotheses is to directly relate those observable events on the skin surface that are closely related to the shape of the object (i.e., skin displacement and its spatial and time derivatives) to the discharge rate of an afferent fiber without considering the intervening mechanisms, such as the stresses and strains at the receptor site.

Discharge rates of SAs to skin indentation with probes (e.g., Knibestöl, 1975; Pubols and Pubols, 1983) can be consistently interpreted as depending on the velocity and displacement of the most sensitive spot. The edge sensitivity of SAs under gratings (Phillips and Johnson, $1981 \mathrm{a}$; Johansson et al., 1982) points to the hypothesis that they are sensitive to skin curvature change. This was confirmed during both stroking and vertical indentation with sinusoidal steps by the moderate discharge of an SA when the most sensitive spot was under the flat portions of the steps, by its peak discharge when the sharpest portion of the steps was on the spot, and by a very low or lack of response to the concave portions of the steps. In the stroking experiments, the presence of a strong interaction between the effects of skin curvature change and stroke velocity (Fig. 12B, LaMotte and Srinivasan, 1987a) made necessary the hypothesis that discharge rate depended on the rate of change of curvature. The presence of clear pauses-not just reductions of discharge rate-between 
rcgions of high rate, and especially the left pause of low-to-high strokes, where no loss of contact between the step and the skin occurred, could only he explained by the cessation of SA response to "unloading" of curvature (negative curvature rate).

In contrast, the RA responses to indentations by gratings (Phillips and Johnson, 1981a) and sinusoidal steps suggest that RAs have little or no sensitivity to skin curvature change. These responses could be interpreted as being predominantly influenced by the vertical velocity of the most sensitive spot. However, under stroking, the excellent discrimination of the various steps, at all stroke velocities, by the RA burst discharge rate (Fig. 8, LaMotte and Srinivasan, 1987b), despite the absence of sensitivity to curvature change, was interpreted as being due to sensitivity to the rate of curvature change. This hypothesis provided an explanation of the strong interaction between step shape and velocity in stroking experiments, and of the slightly enhanced responsc of some RAs under the sharpest portions of the steps in the indentation experiments.

It should be noted that the descriptive explanations given here can be generalized to any arbitrary stimulus. The indications for shape recognition are that, under vertical indentation, the spatial distribution of the number of impulses in the RA population gives weak information about the deflected shape of the skin surface (see Discussion in LaMotte and Srinivasan, 1987b), while the SAs indicate the locations of sharp regions, as well as the curvature distribution, of this deflected shape very well. The hypotheses stated above can be formulated in a manner conducive to a mathematical representation that can be used for testing the hypotheses with quantitative predictions. Once we have confidence in the relationships between surface stimuli and the resulting responses, the finer intermediate mechanisms governing the stresses and strains at the receptor site can be investigated.

\section{Major features of $S A$ and $R A$ responses that contribute to the discrimination of steps under conditions of stroking and vertical indentation}

The shape of an object applied to the skin can be derived from the spatial distribution of the evoked neural activity in the mechanoreceptor population. Spatial response profiles in which either the rate of discharge (Figs. 3 and 8, LaMotte and Srinivasan, 1987a; Fig. 1, 1987b) or the number of impulses (see Figs. 6 and 10 ) is plotted against the spatial position of the step can be interpreted as indicating the spatial responses of a population of mechanoreceptors. These profiles can be described, and discriminated from one another, in terms of several of their features. For example, the widths of bursts and pauses convey "spatial" information by providing the widths of regions of active and inactive receptor populations, respectively; the burst discharge rate conveys "intensive" information related to the sharpness of the object; there is also the general pattern of discharge rate variation within the region of activity, which may be important in the recognition of shape (e.g., the pattern of "burst-pause-burst" in SA responses to steps stroked from the high to the low side; see LaMotte and Srinivasan, 1987a). In the present scries of papers, only steps of similar shapes were used (each having a sinusoidal curvature distribution), resulting in similar patterns of discharge. For the purpose of determining the possible neural codes for discrimination between the various step pairs, it is therefore enough to consider the spatial and intensive features mentioned above.

The results of human discrimination tests, in which pairs of

\begin{tabular}{|c|c|c|c|c|c|}
\hline \multirow[b]{2}{*}{$\begin{array}{l}\text { Mode of } \\
\text { presentation }\end{array}$} & \multirow{2}{*}{$\begin{array}{l}\text { Human }(\mathrm{HU}) \\
\text { or receptor } \\
\text { type }\end{array}$} & \multicolumn{4}{|c|}{ Step pairs: step nos. and $\Delta W(\mu \mathrm{m})$} \\
\hline & & $\begin{array}{l}3 \text { vs } 4 \\
1808\end{array}$ & $\begin{array}{l}1 \text { vs } 2 \\
745\end{array}$ & $\begin{array}{l}4 \text { vs } 5 \\
96\end{array}$ & $\begin{array}{l}2 \text { vs } 3 \\
35\end{array}$ \\
\hline \multirow{4}{*}{$\begin{array}{l}\text { Stroking, } \\
\text { low to high }\end{array}$} & HU & $5 / 5$ & $5 / 5$ & $4 / 5$ & $2 / 5$ \\
\hline & SA & $\mathrm{R}, \mathrm{BW}$ & $\mathrm{R} ?$ & $\mathrm{R}$ ? & - \\
\hline & RA & $\mathrm{R}$ & $\mathrm{R}$ & $\mathrm{R}$ ? & - \\
\hline & $\mathrm{SA}+\mathrm{RA}$ & $\mathrm{R}, \mathrm{BW}$ & $\mathbf{R}$ & $\mathrm{R}$ ? & - \\
\hline \multirow{4}{*}{$\begin{array}{l}\text { Vertical } \\
\text { indentation }\end{array}$} & HU & $5 / 5$ & $3 / 5$ & $1 / 5$ & $1 / 5$ \\
\hline & SA & PM, MR & PM? & - & - \\
\hline & RA & - & - & - & - \\
\hline & $\mathrm{SA}+\mathrm{RA}$ & PM & - & - & - \\
\hline \multirow{4}{*}{$\begin{array}{l}\text { Stroking, } \\
\text { high to low }\end{array}$} & $\mathrm{HU}$ & $5 / 5$ & $2 / 5$ & $0 / 5$ & $0 / 5$ \\
\hline & SA & R?, PW & - & - & - \\
\hline & $\mathrm{RA}$ & $\mathrm{R}, \mathrm{BW}$ & - & - & - \\
\hline & $\mathrm{SA}+\mathrm{RA}$ & $\mathrm{R}, \mathrm{BW}$ & - & - & - \\
\hline
\end{tabular}

HU, Human (proportions of subjects exhibiting above-threshold discrimination are shown). R, Discharge rate; $\mathrm{BW}$, burst width; PW, pause width; PM, peakminus-minimal number of impulses; $M R$, width of modulatory region.

steps were stroked across or vertically indented into the skin, are summarized in Table 1 . The proportions of subjects tested exhibiting above-threshold discrimination are shown. On the average, the subjects discriminated best under low-to-high stroking (step pairs 3 versus 4,1 versus 2 , and 4 versus 5), moderately well under vertical indentations without stroking (step pairs 3 versus 4 and 1 versus 2), and not as well under high-to-low stroking (only step pair 3 versus 4 ).

The conclusions shown in Table 1 regarding discrimination in the neural responses of RAs and SAs, considered both separately and together, were based on the statistical significance achieved under Newman-Keuls pairwise comparisons. Those differences that almost reached significance are indicated by question marks.

Gross differences: steep versus gradual (step 3 versus 4). All the subjects could discriminate the step pair 3 versus 4 under all the 3 stimulus conditions. The spatial features that could differentiate the steep and gradual steps were, for low-to-high strokes, the SA burst widths and, for high-to-low strokes, SA pause widths and RA burst widths. Clearly, the information about the width of the region of active fibers is improved if the SA and RA burst widths are combined. This combined burst width could discriminate the steep and the gradual steps in both stroke directions. Under vertical indentations, the width of the modulatory region for SAs provided a discriminable spatial feature for the 2 categories of steps.

Intensive features that could discriminate were the SA burst rate for low-to-high strokes and the RA burst rates for both stroke directions. The SA burst rate for high-to-low strokes would probably be able to discriminate step 3 from step 4 with a larger sample, since the discharge rates for steps 1 and 2 were significantly different from those for 4 and 5 , and all SAs except one had a higher rate for step 3 than for step 4 . When the SA and RA burst rates were combined, they could discriminate steep steps from the gradual ones for both stroke directions. Under vertical indentations, the intensive measures of $(P-M)$ and $(P-B)$ impulses for SA responses, as well as for combined $\mathrm{SA}$ and RA responses, could discriminate the 2 step categories. Only the peak-minus-minimum results are given in the table. 
Among the various spatial and intensive features that could account for the discrimination between the steep and gradual steps, the spatial cues are perhaps more reliable given the usual variations in velocity and overall force that occur during active touch. It is interesting that among SA and RA burst rates and burst widths under stroking, each receptor type provides a discriminable cue where the other fails (except for low-to-high burst rates, where both receptor types discriminate). When all 3 modes of presentation of steps are viewed together, the SA spatial features emerge as the strongest candidates in the discrimination of steep from gradual steps in the presence of noise due to variations in stimulus parameters.

Fine differences within the categories of steep and gradual steps ( 1 versus 2,2 versus 3 , and 4 versus 5). All the subjects could discriminate the steep steps 1 versus 2 for low-to-high strokes. The corresponding RA burst rates were able to discriminate these steps. The SA burst rates were significantly different for step 1 versus 3 , and all but one SA had higher burst rates for step 1 than for step 2, indicating that, with a bigger sample of fibers, burst rate differences for step 1 versus 2 for low-to-high strokes might achieve statistical significance. Indeed, the combined SA and RA burst rates did discriminate these steps.

In contrast to the gross discrimination of steep versus gradual steps, no spatial features, whether considered separately in the $\mathrm{SA}$ and RA responses, or in the combined response, could discriminate among the steep or the gradual steps under any of the stimulus conditions.

Of the remaining stimulus pairs, the subjects could discriminate step 4 from 5 under low-to-high stroking (4 out of 5 subjects) and step 1 versus 2 under vertical indentation ( 3 out of 5 subjects). The inability of some of the subjects to discriminate these step pairs indicates that the cues must be weak. The mean $\mathrm{SA}$, as well as RA, response measures, both spatial and intensive, did not discriminate these step pairs. Individual SA and RA response measures indicate that it is more likely the intensive features in the responses of the more sensitive members of both RA and SA populations that enable such fine discrimination. In fact, for low-to-high strokes, all but $1 \mathrm{SA}$ and 2 RAs had higher burst rates for step 4 than for step 5 .

It is clear from the above discussion that both spatial and intensive information are available in the responses of the SA or RA population, or both, for discriminations of gross differences in shapes, while only intensive cues are available for finer discriminations. Accuracy of information about the width of the region of active mechanoreceptive afferents is improved when RA and SA responses are combined, as was indicated by the successful discrimination of step 3 versus 4 by the combined burst width for both stroke directions. Combined intensive measures could, in addition, account for the fincr human discrimination of step 1 versus 2 for low-to-high stroking. It is likely that the sensation of width is directly related to the spatial measures, the sensation of sharpness to the intensive measures, and that the subjects principally used the former for gross discrimination and the latter for finer discrimination.

When the finger is actively stroked over a step, there is considerable variability in both the overall force and the velocity of stroking. Information about the overall force is conveyed by the basal discharge. Also, all the conclusions in Table 1 for SA and RA response measures under stroking apply for all stroke velocities tested. Therefore, even moderate perturbations in velocity and force under active stroking of a step pair will not affect their discrimination. However, confusion between, say, low-to-high stroking of step 5 at $40 \mathrm{~mm} / \mathrm{sec}$ and step 1 at $1 \mathrm{~mm} /$ $\mathrm{sec}$ is possible, since both the intensive and spatial response measures of SAs are unable to discriminate this pair (Fig. $11, B, D, F$, LaMotte and Srinivasan, 1987a). But the RA burst rates (Fig. $5 B$, LaMotte and Srinivasan, 1987b), the gross differences in the speed at which the successive mechanoreceptors are activated, and the variation of discharge rate over space and time would provide many cues contributing to easy discrimination.

\section{Conclusion}

In the present and the 2 preceding papers (LaMotte and Srinivasan, 1987a, b), we described the evoked responses of SAs and RAs to steps of varying shape stroked across or indented into the monkey fingerpad. Under stroking, the direction and the velocity were varied, whereas in the vertical indentation experiments, the steps were indented into the skin at successive positions of the step across the receptive field. The capacities of humans to discriminate the same step stimuli applied to the fingerpad were also measured.

Models of SAs and RAs were proposed to directly relate those observable events on the skin surface, which are closely related to the shape of the object, to the discharge rate of the afferent fiber. It was shown that all the major results under both stroking and vertical indentations could be explained consistently by the models in a simple manner, without considering the intervening mechanisms, such as the stresses and strains within the skin at the receptor site.

Human discrimination of the steps under both stroking and vertical indentation was correlated with certain features of the spatial response profiles of individual SAs and RAs. It was concluded that, under either stroking or vertical indentation, the discrimination of gross differences in shape, namely, the steep steps from the gradual, is based primarily on the spatial differences in the widths of regions of active and inactive SAs. Finer discrimination between steps with smaller differences in curvature or sharpness is based mainly on changes in the discharge rate of RAs for stroking and probably of SAs for vertical indentation. Thus, in the recognition and discrimination of shape, predominantly SAs, and, to a lesser extent, RAs provide important information: Under vertical indentation, the SAs are clearly superior in providing both spatial and intensive features; under stroking, RAs provide better intensive information, while the spatial information is conveyed by the SAs.

\section{References}

Darian-Smith, I., and P. Kenins (1980) Innervation density of mechanoreceptive fibres supplying glabrous skin of the monkcy's index finger. J. Physiol. (Lond.) 309: 147-155.

Darian-Smith, I., and L. E. Oke (1980) Peripheral neural representation of the spatial frequency of a grating moving across the monkey's finger pad. J. Physiol. (Lond.) 309: 117-133.

Darian-Smith, I., I. Davidson, and K. O. Johnson (1980) Peripheral neural representation of spatial dimensions of a textured surface moving across the monkey's finger pad. J. Physiol. (Lond.) 309: 135-146.

Johansson, R. S., and A. B. Vallbo (1979) Tactile sensibility in the human hand: Relative and absolute densities of four types of mechanoreceptive units in glabrous skin. J. Physiol. (Lond.) 286: 283-300.

Johansson, R. S., U. Landström, and R. Lundström (1982) Sensitivity to edges of mechanoreceptive afferent units innervating the glabrous skin of the human hand. Brain Res. 244: 27-32.

Johnson, K. O. (1980) Sensory discrimination: Decision process. J. Neurophysiol. 43: 1771-1792.

Johnson, K. O., and G. D. Lamb (1981) Neural mechanisms of spatial 
tactile discrimination: Neural patterns evoked by Braille-like dot patterns in the monkey. J. Physiol. (Lond.) 310: 117-144.

Johnson, K. O., and J. R. Phillips (1981) Tactile spatial resolution. I. Two-point discrimination, gap detection, grating resolution, and letter recognition. J. Neurophysiol. 46: 1177-1191.

Knibestöl, M. (1973) Stimulus-response functions of rapidly adapting mechanoreceptors in the human glabrous skin area. J. Physiol. (Lond.) 232: 427-452

Knibestöl, M. (1975) Stimulus-response functions of slowly adapting mechanoreceptors in the human glabrous skin area. J. Physiol. (Lond.) 245: 63-80.

Lamb, G. D. (1983) Tactile discrimination of texturcd surfaces: Peripheral neural coding in the monkey. J. Physiol. (Lond.) 338: 567587.

LaMotte, R. H., and M. A. Srinivasan (1987a) Tactile discrimination of shape: Responses of slowly adapting mechanoreceptive afferents to a step stroked across the monkey fingerpad. J. Neurosci. 7: 16551671 .

LaMotte, R. H., and M. A. Srinivasan (1987b) Tactile discrimination of shape: Responses of rapidly adapting mechanoreceptive afferents to a step stroked across the monkey fingerpad. J. Neurosci. 7: 16721681 .

LaMotte, R. H., G. M. Whitehouse, C. J. Robinson, and F. Davis (1983) A tactile stimulator for controlled movements of textured surfaces across the skin. J. Electrophysiol. Techniques 10: 1-17.

Phillips, J. R., and K. O. Johnson (1981a) Tactile spatial resolution. II. Neural representation of bars, edges, and gratings in monkey primary afferents. J. Neurophysiol. 46: 1192-1203.
Phillips, J. R., and K. O. Johnson (1981b) Tactile spatial resolution. III. A continuum mechanics model of skin predicting mechanoreceptor responses to bars, edges, and gratings. J. Neurophysiol. 46: 1204-1225.

Pubols, B. H., Jr. (1980) On-versus off-responses of raccoon glabrous skin rapidly adapting cutaneous mechanoreceptors. J. Neurophysiol. 43: $1558-1570$.

Pubols, B. H., Jr. (1982a) Factors affecting cutaneous mechanoreceptor response. I. Constant force versus constant displacement stimulation. J. Neurophysiol. 47: 515-529.

Pubols, B. H., Jr. (1982b) Factors affecting cutaneous mechanoreceptor response. II. Changes in mechanical properties of skin with repeated stimulation. J. Neurophysiol. 47: 530-542.

Pubols, B. H., Jr., and L. M. Pubols (1983) Tactile receptor discharge and mechanical properties of glabrous skin. Fed. Proc. 42: 25282535.

Talbot, W. H., I. Darian-Smith, H. H. Kornhuber, and V. B. Mountcastle (1968) The sense of flutter-vibration: Comparison of the human capacity with response patterns of mechanoreceptive afferents from the monkey hand. J. Neurophysiol. 31: 301-334.

Vierck, C. J. (1979) Comparison of punctate, edge and surface stimulation of peripheral, slowly-adapting, cutaneous, afferent units of cat. Brain Res. 175: 155-159.

Winer, B. J. (1971) Statistical Principles in Experimental Design, McGraw-Hill, New York. 